\title{
Wave drift damping of floating bodies in slow yaw-motion
}

\author{
by \\ John Grue and Enok Palm \\ Mechanics Division, Department of Mathematics, \\ University of Oslo, Norway
}

\begin{abstract}
Wave diffraction, wave forces and wave drift damping due to a floating body performing a slow rotation about the vertical axis (yaw) is considered. The rotation angle of the body may be arbitrary. The angular velocity is assumed small compared to the wave frequency, however. The problem is formulated in the frame of reference following the slow rotation of the body, accounting for the fictive forces. By applying the method of multiple time-scales, the fluid flow is determined consistently to leading order in the slow angular velocity and to second order in the wave amplitude. Mathematical solution of the problem is obtained by means of integral equations being suitable for numerical implementation. The wave forces and the wave drift damping is found by applying conservation of linear and angular momentum. Numerical results show that the wave drift damping coefficient due to a slow yaw-motion of a floating body is one order of magnitude larger than the time-averaged moment when there is no rotation. Thus, wave drift damping is pronounced for resonant low-frequency oscillations about the vertical axis of moored floating bodies. The method is applicable to geometries of arbitrary shape.
\end{abstract}

\section{Introduction}

The induced forces and motions of floating bodies in ocean waves are topics of considerable interest both from a practical and fundamental point of view. Within deep sea technology, for example, new floating production systems like moored ships and small floating oilplatforms are under development for operations in very deep water. The actual water depths are as large as $1000 m-1500 m$, which is true for the oilresources at Vøringsbassenget and Mørebassenget in the Norwegian Sea, but water depths down to $2000 \mathrm{~m}$ are also considered. Accurate computations of wave loads and wave-induced motions of the floating parts of the concept are crucial for the construction and dimensioning of the mooring system, and for the positioning and operation of the whole structure. There are several other examples relating to offshore activity; towing operations of large bodies, manouvering of ships, motions of a body drifting in waves.

While the linear part of the wave forces oscillates with the frequencies of the incoming waves, nonlinear effects give rise to sum- and difference-frequency forces acting on the moored body. The difference-frequency forces may give rise to resonant slowly varying oscillations of the body in the horizontal plane, which may have quite large amplitudes, being determined by the difference-frequency loading, the mass-spring characteristics of 
the body and the moorings, and by the damping forces. We shall in this contribution study wave drift damping, which has proved to be an important damping force of such resonant slowly varying motions, where damping due to linear wave scattering is negligible and viscous forces may be small. Wave drift damping is proportional to the square of the incoming wave amplitude and proportional to the slowly varying velocity of the body.

If the body performs a slow horizontal translation with speed $U$ in the incoming waves, the time-averaged force along the speed direction, $F_{x}$, being proportional to the wave amplitude squared, is a function of the forward speed. If $U$ is small compared to the phase velocity $g / \omega$ of the incoming waves, assuming deep water, where $\omega$ denotes the wave frequency measured in an absolute frame of reference, and $g$ the acceleration due to gravity, the force may be expanded by

$$
F_{x}(U)=F_{x 0}-B_{11} U \omega / g
$$

Here, $F_{x 0}$ denotes the force for $U=0$, and $-B_{11} U \omega / g$ the wave drift damping force. The expansion (1) was first suggested by Wichers and van Sluijs (1979), who studied model tests of the damping of low-frequency oscillations of moored ships, finding a pronounced effect of the wave drift damping. For a body performing a slow horizontal translation with speed $U$ along the $x$-axis, speed $V$ along the $y$-axis, and a slow rotation with angular velocity $\Omega$ about the vertical $z$-axis, with time-averaged horizontal force components $F_{x}$ and $F_{y}$ along the $x$ - and $y$-axes, respectively, and time-averaged moment about the vertical axis, $M_{z}, O-x y z$ a cartesian frame of reference, the generalization of (1) reads

$$
\left(\begin{array}{c}
F_{x} \\
F_{y} \\
M_{z}
\end{array}\right)=\left(\begin{array}{c}
F_{x 0} \\
F_{y 0} \\
M_{z 0}
\end{array}\right)-\left(\begin{array}{lll}
B_{11} & B_{12} & B_{16} \\
B_{21} & B_{22} & B_{26} \\
B_{61} & B_{62} & B_{66}
\end{array}\right)\left(\begin{array}{c}
U \omega / g \\
V \omega / g \\
\Omega / \omega
\end{array}\right)
$$

Here, $\left(F_{x 0}, F_{y 0}, M_{z 0}\right)=\left(F_{x}, F_{y}, M_{z}\right)$ for $U=V=\Omega=0$, and $\left\{B_{i j}\right\}$ denotes the wave drift damping matrix. In the recent years several methods are published for predicting the forces due to a body moving in translatory motion in waves, or a body being stationary in waves and a current, see e.g. Grue \& Palm $(1985,1986)$ for the two-dimensional case, and in three dimensions Huismans \& Hermans (1985), Huismans (1986), Zhao, Faltinsen, Krokstad \& Aanesland (1988), Zhao \& Faltinsen (1989), Nossen, Grue \& Palm (1991), Emmerhoff \& Sclavounos (1992), Grue \& Biberg (1993). These works describe methods to evaluate, among others, the wave drift damping coefficients $B_{11}, B_{12}, B_{21}, B_{22}$.

The theory was extended by Grue \& Palm (1993) to account for the time-averaged moment $M_{z}$ due to a translating body, allowing for predictions of $B_{61}$ and $B_{62}$. They found, among others, that time-averaged velocities in the fluid, being proportional to the wave amplitude squared, gave rise to important contributions to $M_{z}, B_{61}, B_{62}$. At the same time a method for obtaining the complete wave drift damping matrix, and thereby for the first time the damping coefficients $B_{16}, B_{26}, B_{66}$, was given by Newman (1993). He applied a perturbation approach where the low-frequency oscillations of the floating body, which were assumed small, were superposed on the diffraction field. The theoretical framework was given without numerical examples. In a recent work by Sclavounos (1994) the equation governing the slow drift motion of a floating body, including the role of the wave drift damping matrix, was discussed. 
In the present work a method is derived where the main goal is to evaluate the coefficients $B_{i 6}, i=1,2,6$, of the wave drift damping matrix. A floating body performing a slow rotation about the vertical axis while being exposed to incoming monochromatic waves is considered. The rotation angle of the body may be arbitrary. The angular velocity, being a function of time, is assumed to be small compared to the wave frequency of the incoming waves, i.e. $\Omega / \omega<<1$, however, which justifies application of the method of multiple time-scales. The resulting mathematical problem, the fluid flow, and the fluid forces, are then functions of the instantaneous rotation angle of the body.

The mathematical problem is formulated in the frame of reference rotating with the slow angular motion of the body, hereafter denoted by the relative frame of reference, accounting for the fictive forces. The fluid is assumed to be homogenous and incompressible, and viscous effects are disregarded, such that potential theory may be applied. First an exact relation for the fluid pressure in the relative frame of reference is derived. The boundary value problem for the velocity potential is then developed. The method of multiple time-scales, and perturbation expansion of the potential after the wave amplitude and the slow angular velocity are then applied. Next integral equations are derived for the set of potentials, involving unknown quantities on the wetted body surface only. The integral equations are suitable for solution by means of a low-order panel method, which is applied here, giving efficient and robust numerical algoritms. The method is general and is applicable to bodies of arbitrary shape. To simplify the analysis, linear diffraction effects are accounted for in this work. Thus, in the relative frame of reference the body is restrained. The water depth is assumed infinite, and the body is assumed to be wall-sided at the water line.

The time-averaged horizontal force and vertical moment may be obtained in different ways. The most usual procedures are either by integrating the pressure over the instantaneous wetted part of the body surface, or by applying conservation of linear and angular momentum. The latter method is applied here, giving as final result that $F_{x}, F_{y}, M_{z}$, $B_{16}, B_{26}$, and $B_{66}$ are expressed in terms of the far-field amplitudes of the components of the fluid flow.

A code for the complete method is developed, and numerical results are presented for two practical geometries, viz. a ship and a vertical circular cylinder moving with its axis describing a circular path about the origin. We shall find that $B_{16}, B_{26}, B_{66}$, may be one order of magnitude larger than $F_{x 0}, F_{y 0}, M_{z 0}$. For a ship in quartering seas with a wave length being about half of the ship length, for example, it is obtained that $B_{66} / M_{z 0} \simeq 200$. Thus, if the slow angular velocity is $5 \%$ of the wave frequency, the value of $(\Omega / \omega) B_{66}$ is still 10 times larger than $M_{z 0}$ in this case, which means that the damping due to $B_{66}$ is considerable.

The paper is organized as follows: In Section 2 the equation of motion for the fluid in the relative frame of reference is formulated, and an exact relation for the pressure is derived. Furthermore, the fast and the slow time-scales are introduced. In Section 3 the boundary value problem is discussed and the perturbation potentials introduced. In Section 4 the resulting set of boundary value problems are solved by means of integral equations. In Section 5 the analysis for the slowly rotating body is applied to develop integral equations for the velocity potentials due to a translating body in incoming waves. In Sections 
6 and 7 expressions for the damping moment and the damping force, respectively, are derived. Section 8 is devoted to the balance of energy, and Section 9 describes numerical implementation and results. Finally, Section 10 is concluding remarks.

\section{The equation of motion and the pressure}

We consider a floating body performing a slow time-dependent rotation about the vertical axis while being exposed to incoming waves. Two frames of references are introduced, one absolute frame of reference, $O-x^{0} y^{0} z^{0}$, being fixed in space, and one relative frame of reference, $O-x y z$, following the slow rotation of the body. The vertical axis is defined by the $z=z^{0}$-axis, pointing upwards, with $z=z^{0}=0$ coinciding with the mean free surface. Unit vectors $\mathbf{i}, \mathbf{j}, \mathbf{k}$ are introduced along the $x-, y$-,z-axes, respectively. Let $\alpha(t)$ denote the rotation angle of the body relative to the $x^{0}$-axis, and $\boldsymbol{\Omega}=\Omega \mathbf{k}=\dot{\alpha} \mathbf{k}$ the angular velocity, where a dot denotes derivative with respect to time. $O-x^{0} y^{0} z^{0}$ and $O-x y z$ are then related by

$$
\begin{aligned}
& x=x^{0} \cos \alpha+y^{0} \sin \alpha \\
& y=y^{0} \cos \alpha-x^{0} \sin \alpha \\
& z=z^{0}
\end{aligned}
$$

The fluid motion is considered in the relative frame of reference. Neglecting viscous effects the equation of motion for the fluid reads

$$
\rho \frac{\partial \mathbf{v}}{\partial t}+\rho \mathbf{v} \cdot \nabla \mathbf{v}=-\nabla p-\rho g \nabla z+\mathbf{H}
$$

where $\mathbf{v}$ and $p$ denote the velocity and pressure of the fluid, respectively, $\rho$, denotes the density, assumed constant, $g$ denotes the acceleration due to gravity, and $\mathbf{H}$ is given by

$$
\mathbf{H}=-2 \rho \boldsymbol{\Omega} \times \mathbf{v}-\rho \boldsymbol{\Omega} \times \boldsymbol{\Omega} \times \boldsymbol{x}-\rho \dot{\boldsymbol{\Omega}} \times \boldsymbol{x}
$$

Here, the first term denotes the the Coriolis force, the second term the centrifugal force, and the third term the fictive force due to the angular acceleration.

A velocity field given by $-\Omega \times \boldsymbol{x}$ is introduced when observing the fluid velocity from the relative frame of reference. Thus, $\mathbf{v}$ may be decomposed by $\mathbf{v}=\mathbf{v}^{\prime}-\boldsymbol{\Omega} \times \boldsymbol{x}$. Assuming that $\mathbf{v}^{\prime}$ is irrotational, this velocity may be obtained by the gradient of a velocity potential $\Phi^{\prime}$, i.e. $\mathbf{v}^{\prime}=\nabla \Phi^{\prime}$. The equation of motion may then be written in the form

$$
\nabla\left(\frac{\partial \Phi^{\prime}}{\partial t}-\Omega \frac{\partial \Phi^{\prime}}{\partial \theta}+\frac{1}{2}\left|\nabla \Phi^{\prime}\right|^{2}\right)=\nabla\left(-\frac{p}{\rho}-g z\right)
$$

where polar coordinates are introduced by $x=R \cos \theta, y=R \sin \theta$. By integration we obtain the following expression for the fluid pressure

$$
-\frac{p}{\rho}=\frac{\partial \Phi^{\prime}}{\partial t}-\Omega \frac{\partial \Phi^{\prime}}{\partial \theta}+\frac{1}{2}\left|\nabla \Phi^{\prime}\right|^{2}+g z+C(t)
$$

where $C(t)$ is an arbitrary function of time. Both (8) and (9) are exact. 


\subsection{Fast and slow time-scales}

The wave number of the incoming waves, $K$, nondimensionalized by the characteristic length of the floating body, $l$, is assumed to be of order unity. For deep water waves this means that $\omega^{2} l / g=O(1)$, where $\omega$ denotes the wave frequency in the absolute frame of reference. The rotation angle of the body may be of arbitrary magnitude, i.e. $\alpha(t)=O(1)$. The angular velocity $\Omega$ is, however, assumed to be much smaller than the wave frequency $\omega$, i.e.

$$
\Omega / \omega<<1
$$

Since the rotation angle is finite, this assumption implies that

$$
\dot{\Omega} / \omega^{2}=O\left(\Omega^{2} / \omega^{2}\right)<<\Omega / \omega
$$

In the following analysis we shall apply a perturbation expansion in $\Omega / \omega$, retaining terms up to order $\Omega / \omega$. This means that the perturbed problem has two time-scales, a fast time-scale with characteristic time $1 / \omega$, and a slow time-scale with characteristic time $1 / \Omega$. In obtaining e.g. the wave drift damping force and moment a time-average over the fast time-scale is applied.

\section{The boundary value problems}

Assuming that the fluid is incompressible, $\Phi^{\prime}$ satisfies the Laplace equation in the fluid domain. It is convenient to decompose $\Phi^{\prime}$ by

$$
\Phi^{\prime}=\phi_{s}+\Phi+\psi^{(2)}
$$

where $\phi_{s} \equiv \Omega \chi_{6}$ denotes the potential generated by the body when there are no waves, $\Phi$ the linear wave potential being proportional to the amplitude $A$ of the incoming waves, and $\psi^{(2)}$ a time-averaged second order potential being proportional to the wave amplitude squared.

\subsection{Steady potentials $\chi_{1}, \chi_{2}, \chi_{6}$}

The steady potential $\chi_{6}$ appears in (12). Later, potentials $\chi_{1}$ and $\chi_{2}$ will also be required. The potentials $\chi_{i}, i=1,2,6$, satisfy the following boundary value problems

$$
\begin{aligned}
& \nabla^{2} \chi_{i}=0 \quad \text { in the fluid domain } \\
& \frac{\partial \chi_{i}}{\partial z}=0 \quad \text { at } \quad z=0 \\
& \frac{\partial \chi_{i}}{\partial n}=n_{i} \quad \text { at } \quad S_{B} \\
& \left|\nabla \chi_{i}\right| \rightarrow 0 \quad R \rightarrow \infty, \quad z \rightarrow-\infty
\end{aligned}
$$

where $\mathbf{n}=\left(n_{1}, n_{2}, n_{3}\right)$ denotes the unit normal at $S_{B}$, pointing out of the fluid, and $n_{6}=\mathbf{n} \cdot(\mathbf{k} \times \boldsymbol{x})=n_{2} x-n_{1} y$. The potentials $\chi_{i}, i=1,2,6$, are obtained by means of source distributions. 


\subsection{The boundary conditions, $\Phi$}

To obtain the free surface condition for $\Phi$, the individual time-derivative is applied to (9) at $z=\zeta$. After linearizing with respect to the wave amplitude we find

$$
\frac{\partial^{2} \Phi}{\partial t^{2}}-2 \Omega \frac{\partial^{2} \Phi}{\partial \theta \partial t}+2 \nabla_{h} \phi_{s} \cdot \nabla_{h} \frac{\partial \Phi}{\partial t}-\frac{\partial^{2} \phi_{s}}{\partial z^{2}} \frac{\partial \Phi}{\partial t}+g \frac{\partial \Phi}{\partial z}=0 \quad \text { at } \quad z=0
$$

where $\nabla_{h}$ denotes the horizontal gradient. Let us then introduce $\Phi=\operatorname{Re}\left[(A i g / \omega) \phi e^{i \omega t}\right]$. Noting that $\Phi$ and $\phi$ are functions of $\alpha, \Omega, \dot{\Omega}, \ldots$, we find for the partial time-derivative of $\Phi$

$$
\frac{\partial \Phi}{\partial t}=R e\left\{\frac{A i g}{\omega}\left(i \omega \phi+\Omega \frac{\partial \phi}{\partial \alpha}+\dot{\Omega} \frac{\partial \phi}{\partial \Omega}+\ldots\right) e^{i \omega t}\right\}
$$

We now introduce the perturbation parameter $\epsilon \equiv \Omega / \omega$. By expanding (14) after $\epsilon$, retaining terms up to $O(\epsilon)$, the free surface condition for $\phi$ reads

$$
-K \phi+2 i \epsilon K \frac{\partial \phi}{\partial \alpha}-2 i \epsilon K \frac{\partial \phi}{\partial \theta}+2 i \epsilon K \nabla \phi \cdot \nabla_{h} \chi_{6}+i \epsilon K \phi \nabla_{h}^{2} \chi_{6}+\frac{\partial \phi}{\partial z}=0 \quad \text { at } \quad z=0
$$

In this paper we consider the diffraction problem, which means that the body has no motions in the relative frame of reference. The boundary condition for $\phi$ at the body is then given by

$$
\frac{\partial \phi}{\partial n}=0 \quad \text { at } \quad S_{B}
$$

\subsection{Perturbation procedure}

It is convenient to expand the potential $\phi$ in $\epsilon$ by

$$
\phi=\phi^{0}+\epsilon \phi^{1}
$$

$\phi^{0}$ then satisfies

$$
\begin{gathered}
-K \phi^{0}+\frac{\partial \phi^{0}}{\partial z}=0 \text { at } z=0 \\
\frac{\partial \phi^{0}}{\partial n}=0 \quad \text { at } S_{B}
\end{gathered}
$$

$\phi^{0}$ is composed by the incoming wave potential, $\phi_{I}$, and the scattering potential, $\phi_{7}$, i.e. $\phi^{0}=\phi_{I}+\phi_{7}$. In addition to the conditions at the free surface and the body boundary, $\phi_{7}$ satisfies $\left|\nabla \phi_{7}\right| \rightarrow 0$ for $z \rightarrow-\infty$ and the radiation condition for $R \rightarrow \infty$, i.e.

$$
\phi_{7}=R^{-1 / 2} H^{0}(\theta) e^{K z-i K R}
$$

where $H^{0}(\theta)$ denotes the far-field amplitude of the potential, which is determined by (102).

The incoming wave potential is given by, assuming water of infinite depth,

$$
\phi_{I}=e^{K z-i K R \cos (\beta-\theta)}
$$


where $K=\omega^{2} / g$, and $\beta$ denotes the angle between the $x$-axis and the wave direction (in the relative frame of reference). $\beta$ is related to the angle between the $x^{0}$-direction and the wave direction, $\beta^{0}$, (in the absolute frame of reference) by $\beta=\beta^{0}-\alpha$. It is obvious that $\phi^{0}$ is a function of $\beta=\beta^{0}-\alpha$. This means that $\partial \phi^{0} / \partial \alpha=-\partial \phi^{0} / \partial \beta$. The free surface boundary condition for $\phi^{1}$ then becomes

$$
-K \phi^{1}+\frac{\partial \phi^{1}}{\partial z}=2 i K \frac{\partial \phi^{0}}{\partial \beta}+2 i K \frac{\partial \phi^{0}}{\partial \theta}-2 i K \nabla_{h} \phi^{0} \cdot \nabla_{h} \chi_{6}-i K \phi^{0} \nabla_{h}^{2} \chi_{6} \quad \text { at } \quad z=0
$$

In the diffraction problem the body boundary condition is

$$
\frac{\partial \phi^{1}}{\partial n}=0 \quad \text { at } \quad S_{B}
$$

In addition, $\phi^{1}$ satisfies the condition of outgoing waves as $R \rightarrow \infty$, and $\left|\nabla \phi^{1}\right| \rightarrow 0$ for $z \rightarrow-\infty$.

It is convenient to decompose the boundary value problem for $\phi^{1}$ by introducing

$$
\phi^{1}=\phi^{11}+\phi^{12}+\phi^{13}
$$

where $\phi^{11}, \phi^{12}, \phi^{13}$ satisfy the following set of boundary value problems

$$
\begin{gathered}
-K \phi^{11}+\frac{\partial \phi^{11}}{\partial z}=2 i K \frac{\partial \phi^{0}}{\partial \beta} \quad \text { at } z=0 \\
\frac{\partial \phi^{11}}{\partial n}=0 \quad \text { at } S_{B} \\
-K \phi^{12}+\frac{\partial \phi^{12}}{\partial z}=2 i K \frac{\partial \phi^{0}}{\partial \theta} \quad \text { at } z=0 \\
-K \phi^{13}+\frac{\partial \phi^{13}}{\partial z}=-2 i K \nabla_{h} \phi^{0} \cdot \nabla_{h} \chi_{6}-i K \phi^{0} \nabla_{h}^{2} \chi_{6} \text { at } z=0 \\
\frac{\partial \phi^{13}}{\partial n}=-\frac{\partial \phi^{12}}{\partial n} \text { at } S_{B}
\end{gathered}
$$

The potentials $\phi^{11}$ and $\phi^{12}$ are parts of the solution of the boundary value problem for $\phi^{1}$, and may be expressed in terms of $\phi^{0}$ by

$$
\begin{aligned}
\phi^{11} & =2 i K \frac{\partial^{2} \phi^{0}}{\partial \beta \partial K} \\
\phi^{12} & =2 i K \frac{\partial^{2} \phi^{0}}{\partial \theta \partial K}
\end{aligned}
$$

$\phi^{13}$ satisfies, in addition to (29) - (30), $\left|\nabla \phi^{13}\right| \rightarrow 0$ for $z \rightarrow-\infty$, and the radiation condition for $R \rightarrow \infty$, i.e.

$$
\phi^{13}=R^{-1 / 2} H^{13}(\theta) e^{K z-i K R}
$$

where $H^{13}(\theta)$ denotes the far-field amplitude of the potential, which is determined by (104). 


\subsection{The boundary conditions, $\psi^{(2)}$}

The second order potential $\psi^{(2)}$ appears in the formulae for the second order fluid pressure, and for the mean force and moment always multiplied by the slow angular velocity $\Omega$. To leading order in $\Omega$ it is then sufficient to consider the boundary value problem for $\psi^{(2)}$ when $\Omega=0$. The free surface condition for $\psi^{(2)}$ then reads

$$
\frac{\partial \psi^{(2)}}{\partial z}=-\frac{1}{g} \overline{\frac{\partial}{\partial t} \nabla \Phi \cdot \nabla \Phi}+\frac{1}{g^{2}} \overline{\frac{\partial \Phi}{\partial t} \frac{\partial^{3} \Phi}{\partial z \partial t^{2}}}+\frac{1}{g} \frac{\overline{\partial \Phi}}{\partial t} \frac{\partial^{2} \Phi}{\partial z^{2}}=-\frac{A^{2} g}{2 \omega} \operatorname{Im}\left(\phi^{0} \frac{\partial^{2} \phi^{0 *}}{\partial z^{2}}\right) \quad \text { at } z=0
$$

where a bar denotes time-average and a star complex conjungate. In the diffraction problem, $\partial \psi^{(2)} / \partial n=0$ at the body surface. In addition, $\left|\nabla \psi^{(2)}\right| \rightarrow 0$ for $R \rightarrow \infty$, or $z \rightarrow-\infty$.

The solution for $\psi^{(2)}$ may easily be obtained by a source distribution. The analysis below shows, however, that only the boundary conditions for $\psi^{(2)}$ are required to find the mean force and moment acting on the floating body. Thus, the complete solution for $\psi^{(2)}$ is not needed. In the general case $\psi^{(2)}$ also satisfies a nontrivial boundary condition at the body boundary, see Grue and Palm (1993, eqs. 11-12). In that paper a discussion of the significance of $\psi^{(2)}$ is given.

\section{$4 \quad$ Integral equations}

The velocity potential $\phi^{0}$ is most easily found by deriving an integral equation, as shown below. When $\phi^{0}$ is known, $\phi^{11}$ and $\phi^{12}$ are then given by (31) and (32), respectively. These formulae are, however, not in a proper form for numerical use for a general body. We shall therefore in this section derive integral equations for $\phi^{11}$ and $\phi^{12}+\phi^{13}=\phi^{1}-\phi^{11}$. By adding these equations we obtain an integral equation for $\phi^{1}$.

\subsection{The potential $\phi^{0}$}

To solve the boundary value problem for $\phi^{0}$ we first introduce a Green function, $G^{0}(a, b, c, x, y, z)$, being a sink at $\boldsymbol{x}=\boldsymbol{a}=(a, b, c)$, satisfying the free surface boundary condition (19). This Green function may be written by, see e.g. Wehausen and Laitone (1960, eq. 13.17),

$$
G^{0}=\frac{1}{r}+\int_{0}^{\infty} \frac{k+K}{k-K} e^{k(z+c)} J_{0}\left(k R^{\prime}\right) d k
$$

where $r=|\boldsymbol{x}-\boldsymbol{a}|, R^{\prime}=\sqrt{(x-a)^{2}+(y-b)^{2}}, J_{0}$ denotes the Bessel function of first kind and order zero, and the path of integration is above the pole at $k=K$. For $R \rightarrow \infty, G^{0}$ takes the form

$$
G^{0}=R^{-1 / 2} h^{0}(\theta) e^{K z-i K R}
$$

where

$$
h^{0}=(8 \pi K)^{\frac{1}{2}} \exp [K(c+i a \cos \theta+i b \sin \theta)-i \pi / 4]
$$


By applying Green's theorem to $\phi^{0}$ and $G^{0}$ it may be shown that $\phi^{0}$ satisfies, see e.g. Nossen, Grue and Palm (1991, eq. 40),

$$
\int_{S_{B}} \phi^{0} \frac{\partial G^{0}}{\partial n} d S-4 \pi \phi_{I}=\left\{\begin{array}{cc}
-2 \pi \phi^{0}(\boldsymbol{x}) & \boldsymbol{x} \in S_{B} \\
-4 \pi \phi^{0}(\boldsymbol{x}) & \boldsymbol{x} \in \mathcal{V}
\end{array}\right.
$$

where $\mathcal{V}$ denotes the fluid volume enclosed by $S_{B}$, the free surface, $S_{F}$, and the vertical circular cylinder, $S(R)$, with radius $R$. The integration is over the $(a, b, c)$-variables. The first case is an integral equation for $\phi^{0}$.

\subsection{The potential $\phi^{1}$}

To find an integral equation for $\phi^{1}$ we first introduce an auxillary function, $G^{1}(a, b, c, x, y, z)$, being regular for $c<0, z<0$, satisfying the following free surface boundary condition

$$
-K G^{1}+\frac{\partial G^{1}}{\partial c}=2 i K \frac{\partial G^{0}}{\partial \tilde{\theta}} \quad \text { at } \quad c=0
$$

where $\tilde{\theta}$ is defined by $a=\tilde{R} \cos \tilde{\theta}, b=\tilde{R} \sin \tilde{\theta}, \tilde{R}^{2}=a^{2}+b^{2}$. It is easily shown that $G^{1}$ may be expressed in terms of $G^{0}$ by

$$
G^{1}=2 i K \frac{\partial^{2} G^{0}}{\partial \tilde{\theta} \partial K}
$$

First the potential $\phi^{11}$, which is determined by (31) is considered. An integral equation for $\phi^{11}$ is obtained by differentiating the integral equation (38) for $\phi^{0}$ with respect to $\beta$ and $K$. We then obtain

$$
\int_{S_{B}} \phi_{\beta K}^{0} \frac{\partial G^{0}}{\partial n} d S+\int_{S_{B}} \phi_{\beta}^{0} \frac{\partial^{2} G^{0}}{\partial n \partial K} d S-4 \pi \phi_{I, \beta K}=\left\{\begin{array}{cc}
-2 \pi \phi_{\beta K}^{0}(\boldsymbol{x}) & \boldsymbol{x} \in S_{B} \\
-4 \pi \phi_{\beta K}^{0}(\boldsymbol{x}) & \boldsymbol{x} \in \mathcal{V}
\end{array}\right.
$$

where $\phi_{\beta}^{0} \equiv \partial \phi^{0} / \partial \beta, \phi_{\beta K}^{0} \equiv \partial^{2} \phi^{0} / \partial \beta \partial K, \phi_{I, \beta K} \equiv \partial^{2} \phi_{I} / \partial \beta \partial K$. This equation determines $\phi_{\beta K}^{0}$. By then multiplying with $2 i K$, we obtain $\phi^{11}$. A similar procedure may be applied to obtain $\phi^{12}$, and then finally an integral equation for $\phi^{13}$ is derived. However, it is more convenient to derive an integral equation for $\phi^{12}+\phi^{13}$ instead of working with $\phi^{12}$ and $\phi^{13}$ separately. To do so we first apply Green's theorem to $\psi=\phi^{12}+\phi^{13}=\phi^{1}-\phi^{11}$ and $G^{0}$, giving

$$
\int_{S_{B}} \psi \frac{\partial G^{0}}{\partial n} d S+\int_{S_{F}+S(R)}\left(\psi \frac{\partial G^{0}}{\partial n}-G^{0} \frac{\partial \psi}{\partial n}\right) d S=\left\{\begin{array}{cc}
-2 \pi \psi(\boldsymbol{x}) & \boldsymbol{x} \in S_{B} \\
-4 \pi \psi(\boldsymbol{x}) & \boldsymbol{x} \in \mathcal{V}
\end{array}\right.
$$

Now, $\phi^{13} \partial G^{0} / \partial n-G^{0} \partial \phi^{13} / \partial n$ at $S(R)$ vanishes as $R \rightarrow \infty$ since $\phi^{13}$ and $G^{0}$ satisfy the same radiation condition. By then applying the free surface boundary conditions for $\phi^{12}$, $\phi^{13}$ and $G^{0}$, and the body boundary condition for $\psi,(42)$ reduces to

$$
\begin{aligned}
& \int_{S_{B}} \psi \frac{\partial G^{0}}{\partial n} d S+2 i K \int_{S_{F}} G^{0}\left(-\frac{\partial \phi^{0}}{\partial \tilde{\theta}}+\nabla_{h} \phi^{0} \cdot \nabla_{h} \chi_{6}+\frac{1}{2} \phi^{0} \nabla_{h}^{2} \chi_{6}\right) d S \\
& +\int_{S(R)}\left(\phi^{12} \frac{\partial G^{0}}{\partial n}-G^{0} \frac{\partial \phi^{12}}{\partial n}\right) d S=\left\{\begin{array}{cc}
-2 \pi \psi(\boldsymbol{x}) & \boldsymbol{x} \in S_{B} \\
-4 \pi \psi(\boldsymbol{x}) & \boldsymbol{x} \in \mathcal{V}
\end{array}\right.
\end{aligned}
$$


The integrals over $S_{F}$ and $S(R)$ are not on a final form, and may be further developed. To do this we next apply Green's theorem to $\phi^{0}$ and $G^{1}$. By introducing the boundary conditions for $\phi^{0}$ and $G^{1}$ at the free surface we obtain

$$
\int_{S_{B}} \phi^{0} \frac{\partial G^{1}}{\partial n} d S+2 i K \int_{S_{F}} \phi^{0} \frac{\partial G^{0}}{\partial \tilde{\theta}} d S+\int_{S(R)}\left(\phi^{0} \frac{\partial G^{1}}{\partial n}-G^{1} \frac{\partial \phi^{0}}{\partial n}\right) d S=0
$$

Subtracting (44) from (43) gives

$$
\begin{aligned}
& \int_{S_{B}}\left(\psi \frac{\partial G^{0}}{\partial n}-\phi^{0} \frac{\partial G^{1}}{\partial n}\right) d S \\
&+2 i K \int_{S_{F}}\left[-\frac{\partial}{\partial \tilde{\theta}}\left(G^{0} \phi^{0}\right)+G^{0}\left(\nabla_{h} \phi^{0} \cdot \nabla_{h} \chi_{6}+\frac{1}{2} \phi^{0} \nabla_{h}^{2} \chi_{6}\right)\right] d S \\
&+I=\left\{\begin{array}{cc}
-2 \pi \psi(\boldsymbol{x}) & \boldsymbol{x} \in S_{B} \\
-4 \pi \psi(\boldsymbol{x}) & \boldsymbol{x} \in \mathcal{V}
\end{array}\right.
\end{aligned}
$$

where

$$
I=\int_{S(R)}\left(\phi^{12} \frac{\partial G^{0}}{\partial n}-G^{0} \frac{\partial \phi^{12}}{\partial n}-\phi^{0} \frac{\partial G^{1}}{\partial n}+G^{1} \frac{\partial \phi^{0}}{\partial n}\right) d S
$$

It is shown in Appendix A that $I$ equals

$$
I=8 \pi i K \frac{\partial^{2} \phi_{I}}{\partial \beta \partial K}
$$

The free surface integral in (45) may be further developed by noting that

$$
\begin{aligned}
& -\frac{\partial}{\partial \tilde{\theta}}\left(G^{0} \phi^{0}\right)+G^{0}\left(\nabla_{h} \phi^{0} \cdot \nabla_{h} \chi_{6}+\frac{1}{2} \phi^{0} \nabla_{h}^{2} \chi_{6}\right) \\
& \quad=\nabla_{h} \cdot\left[G^{0} \phi^{0}\left(\nabla_{h} \chi_{6}-\mathbf{k} \times \boldsymbol{x}\right)\right]-\phi^{0}\left(\nabla_{h} G^{0} \cdot \nabla_{h} \chi_{6}+\frac{1}{2} G^{0} \nabla_{h}^{2} \chi_{6}\right)
\end{aligned}
$$

By application of Gauss' theorem, assuming that the body is wall-sided at the water line, we then obtain

$$
\begin{aligned}
\int_{S_{F}}\left[-\frac{\partial}{\partial \tilde{\theta}}\left(G^{0} \phi^{0}\right)\right. & \left.+G^{0}\left(\nabla_{h} \phi^{0} \cdot \nabla_{h} \chi_{6}+\frac{1}{2} \phi^{0} \nabla_{h}^{2} \chi_{6}\right)\right] d S \\
=-\int_{S_{F}} & \phi^{0}\left(\nabla_{h} G^{0} \cdot \nabla_{h} \chi_{6}+\frac{1}{2} G^{0} \nabla_{h}^{2} \chi_{6}\right) d S \\
& +\int_{C_{B}+C(R)} \phi^{0} G^{0}\left[\frac{\partial \chi_{6}}{\partial n}-\mathbf{n} \cdot(\mathbf{k} \times \boldsymbol{x})\right] d l
\end{aligned}
$$

where $C_{B}$ and $C(R)$ denote the contours at $z=0$ of $S_{B}$ and $S(R)$, respectively. The integral along $C_{B}$ vanishes due to the boundary condition (13) for $\chi_{6}$ at $S_{B}$. The integrand at $C(R)$ disappears as $R \rightarrow \infty$. The integral over $S_{F}$ is now on a form which converges very rapidly. 


\subsection{The integral equation for $\phi^{1}$}

By then combining (45), (47), (49), we arrive at the following result for $\phi^{1}-\phi^{11}$

$$
\begin{aligned}
& \int_{S_{B}}\left(\phi^{1}-\phi^{11}\right) \frac{\partial G^{0}}{\partial n} d S+8 \pi i K \frac{\partial^{2} \phi_{I}}{\partial \beta \partial K}-\int_{S_{B}} \phi^{0} \frac{\partial G^{1}}{\partial n} d S \\
& -2 i K \int_{S_{F}} \phi^{0}\left(\nabla_{h} G^{0} \cdot \nabla_{h} \chi_{6}+\frac{1}{2} G^{0} \nabla_{h}^{2} \chi_{6}\right) d S=\left\{\begin{array}{cc}
-2 \pi\left(\phi^{1}-\phi^{11}\right)(\boldsymbol{x}) & \boldsymbol{x} \in S_{B} \\
-4 \pi\left(\phi^{1}-\phi^{11}\right)(\boldsymbol{x}) & \boldsymbol{x} \in \mathcal{V}
\end{array}\right.
\end{aligned}
$$

In the first case (50) is an integral equation for $\phi^{1}-\phi^{11}$. We have then obtained (41) and (50) which together determines $\phi^{1}$. By adding (41) multiplied by $2 i K$ and (50) we then obtain the following result for $\phi^{1}$

$$
\begin{aligned}
& \int_{S_{B}} \phi^{1} \frac{\partial G^{0}}{\partial n} d S-\int_{S_{B}}\left(\phi^{0} \frac{\partial G^{1}}{\partial n}+2 i K \frac{\partial \phi^{0}}{\partial \beta} \frac{\partial^{2} G^{0}}{\partial n \partial K}\right) d S \\
& -2 i K \int_{S_{F}} \phi^{0}\left(\nabla_{h} G^{0} \cdot \nabla_{h} \chi_{6}+\frac{1}{2} G^{0} \nabla_{h}^{2} \chi_{6}\right) d S=\left\{\begin{array}{cc}
-2 \pi \phi^{1}(\boldsymbol{x}) & \boldsymbol{x} \in S_{B} \\
-4 \pi \phi^{1}(\boldsymbol{x}) & \boldsymbol{x} \in \mathcal{V}
\end{array}\right.
\end{aligned}
$$

In the first case (51) is an integral equation for $\phi^{1}$. The integral equations for $\phi^{1}$ and $\phi^{1}-\phi^{11}$ are easily solved by means of a panel method. Here a low-order panel method is used as numerical tool. More details about the numerical implementation of the integral equations are described in Section 9.

\section{$5 \quad$ Integral equations due to slow translatory motions}

The present analysis may be applied to derive integral equations for the potentials due to a body translating with a small forward velocity. This may be achieved by situating the body, with finite dimensions, at two different positions in the rotating frame of reference, namely at $y \rightarrow-\infty$ (and $x=0)$, and at $x=\infty$ (and $y=0)$, respectively. In the first case, the rotation of the body with respect to the origin corresponds to a translation along the $x$-direction, while in the second case it corresponds to a translation along the $y$-direction.

\subsection{Translation along the $x$-direction}

Let first the body be situated at a position $y \rightarrow-\infty$, such that $-\Omega y \rightarrow U$, where $U$ is a small velocity along the positive $x$-direction. Then

$$
\Omega \chi_{6} \rightarrow U \chi_{1}, \quad \Omega \frac{\partial}{\partial \theta} \rightarrow U \frac{\partial}{\partial x}, \quad \Omega \frac{\partial \phi^{0}}{\partial \beta} \rightarrow i K U \cos \beta \phi^{0}, \quad \Omega G^{1} \rightarrow-2 i K U \frac{\partial^{2} G^{0}}{\partial x \partial K}
$$

The fluid velocity is then given by $\mathbf{v}=-U \mathbf{i}+\nabla \Phi^{\prime}$, where

$$
\Phi^{\prime}=\operatorname{Re}\left[(A i g / \omega) \phi e^{i \omega t}\right]+U \chi_{1}+\psi^{(2)}
$$

By introducing $\tau(U)=\omega U / g$, the potential $\phi$ is expanded by

$$
\phi=\phi^{0}+\tau(U) \phi^{1 U}
$$


The potential $\phi^{0}$ is determined by (38), $\chi_{1}$ is defined above, and $\psi^{(2)}$ is defined by the boundary value problem formulated in Section 3.4. To obtain the potential $\phi^{1 U}$ we first relate $\phi^{1 U}$ to $\phi^{1}$ by

$$
\frac{\Omega}{\omega} \phi^{1}=\frac{K U}{\omega} \phi^{1 U}=\tau(U) \phi^{1 U}
$$

The boundary condition for $\phi^{1 U}$ at the free surface may then be obtained by introducing (52) and (55) in (23), giving

$$
-K \phi^{1 U}+\frac{\partial \phi^{1 U}}{\partial z}=-2 K \cos \beta \phi^{0}+2 i \frac{\partial \phi^{0}}{\partial x}-2 i \nabla_{h} \phi^{0} \cdot \nabla_{h} \chi_{1}-i \phi^{0} \nabla_{h}^{2} \chi_{1} \quad \text { at } \quad z=0
$$

In addition, $\phi^{1 U}$ satisfies $\partial \phi^{1 U} / \partial n=0$ at the body, $\nabla \phi^{1 U} \rightarrow 0$ for $z \rightarrow-\infty$, and a radiation condition for $R \rightarrow \infty$. The boundary value problem for $\phi^{1 U}$ may be solved by means of an integral equation, which easily is obtained by introducing (52), (55) and (55) into (51), giving

$$
\begin{aligned}
& \int_{S_{B}} \phi^{1 U} \frac{\partial G^{0}}{\partial n} d S-\int_{S_{B}} \phi^{0}\left(2 K \cos \beta \frac{\partial^{2} G^{0}}{\partial n \partial K}-2 i \frac{\partial}{\partial n} \frac{\partial^{2} G^{0}}{\partial x \partial K}\right) d S \\
& -2 i \int_{S_{F}} \phi^{0}\left(\nabla_{h} G^{0} \cdot \nabla_{h} \chi_{1}+\frac{1}{2} G^{0} \nabla_{h}^{2} \chi_{1}\right) d S=\left\{\begin{array}{c}
-2 \pi \phi^{1 U}(\boldsymbol{x}) \\
-4 \pi \phi^{1 U}(\boldsymbol{x}) \boldsymbol{x} \in \mathcal{V}
\end{array}\right.
\end{aligned}
$$

where the first case is an integral equation for $\phi^{1 U}$. Wave radiation and wave diffraction due to a body with a small forward speed was studied by Nossen, Grue and Palm (1991), who applied the decomposition $\phi=\phi^{0}(\nu)+\tau(U) \tilde{\phi}^{1}(\nu)$ of the potential $\phi$, where $\nu=$ $K-2 K \cos \beta \tau(U)$. They arrived at the following integral equation for $\tilde{\phi}^{1}(\nu)$

$$
\begin{aligned}
& \int_{S_{B}} \tilde{\phi}^{1} \frac{\partial G^{0}}{\partial n} d S-\int_{S_{B}} 2 i \phi^{0} \frac{\partial}{\partial n} \frac{\partial^{2} G^{0}}{\partial x \partial \nu} d S \\
& -2 i \int_{S_{F}} \phi^{0}\left(\nabla_{h} G^{0} \cdot \nabla_{h} \chi_{1}+\frac{1}{2} G^{0} \nabla_{h}^{2} \chi_{1}\right) d S=\left\{\begin{array}{cc}
-2 \pi \tilde{\phi}^{1}(\boldsymbol{x}) & \boldsymbol{x} \in S_{B} \\
-4 \pi \tilde{\phi}^{1}(\boldsymbol{x}) & \boldsymbol{x} \in \mathcal{V}
\end{array}\right.
\end{aligned}
$$

Now,

$$
\phi=\phi^{0}(\nu)+\tau(U) \tilde{\phi}^{1}(\nu)=\phi^{0}(K)+\tau(U)\left[\tilde{\phi}^{1}(K)-2 K \cos \beta \phi_{\nu}^{0}(K)\right]+O\left(\tau(U)^{2}\right)
$$

The potential $-2 K \cos \beta \phi_{\nu}^{0}(K)$ may be obtained by the following integral equation

$$
\int_{S_{B}}\left(-2 K \cos \beta \phi_{K}^{0}\right) \frac{\partial G^{0}}{\partial n} d S-\int_{S_{B}} 2 K \cos \beta \phi^{0} \frac{\partial^{2} G^{0}}{\partial n \partial K} d S=\left\{\begin{array}{cc}
-2 \pi \phi^{1 U}(\boldsymbol{x}) & \boldsymbol{x} \in S_{B} \\
-4 \pi \phi^{1 U}(\boldsymbol{x}) & \boldsymbol{x} \in \mathcal{V}
\end{array}\right.
$$

By adding (58), with $\nu$ replaced by $K$, and (60) we obtain the integral equation (57) for $\phi^{1 U}=\tilde{\phi}^{1}(K)-2 K \cos \beta \phi_{\nu}^{0}(K)$, as expected.

\subsection{Translation along the $y$-direction}

Let then the body be situated at a position $x \rightarrow \infty$, such that $\Omega x \rightarrow V$, where $V$ is a small velocity along the $y$-direction. Then,

$$
\Omega \chi_{6} \rightarrow V \chi_{2}, \quad \Omega \frac{\partial}{\partial \theta} \rightarrow V \frac{\partial}{\partial y}, \quad \Omega \frac{\partial \phi^{0}}{\partial \beta} \rightarrow i K V \sin \beta \phi^{0}, \quad \Omega G^{1} \rightarrow-2 i K V \frac{\partial^{2} G^{0}}{\partial y \partial K}
$$


The fluid velocity is then given by $\mathbf{v}=-V \mathbf{j}+\nabla \Phi^{\prime}$, where

$$
\Phi^{\prime}=\operatorname{Re}\left[(A i g / \omega) \phi e^{i \omega t}\right]+V \chi_{2}+\psi^{(2)}
$$

$\phi$ is then expanded by

$$
\phi=\phi^{0}+\tau(V) \phi^{1 V}, \quad \tau(V)=\omega V / g
$$

$\phi^{0}, \chi_{2}$, and $\psi^{(2)}$ are determined as above. To determine $\phi^{1 V}$ we first introduce

$$
\frac{\Omega}{\omega} \phi^{1}=\frac{K V}{\omega} \phi^{1 V}=\tau(V) \phi^{1 V}
$$

Following the same procedure as above we may find the free surface boundary condition for $\phi^{1 V}$ by introducing (61) and (64) in (23), giving

$$
-K \phi^{1 V}+\frac{\partial \phi^{1 V}}{\partial z}=-2 K \sin \beta \phi^{0}+2 i \frac{\partial \phi^{0}}{\partial y}-2 i \nabla_{h} \phi^{0} \cdot \nabla_{h} \chi_{2}-i \phi^{0} \nabla_{h}^{2} \chi_{2} \quad \text { at } \quad z=0
$$

In addition, $\phi^{1 V}$ satisfies $\partial \phi^{1 V} / \partial n=0$ at the body, $\nabla \phi^{1 V} \rightarrow 0$ for $z \rightarrow-\infty$, and a radiation condition for $R \rightarrow \infty$. Introducing (61), (63) and (64) into (51) we then obtain the following integral equation for $\phi^{1 V}$

$$
\begin{aligned}
& \int_{S_{B}} \phi^{1 V} \frac{\partial G^{0}}{\partial n} d S-\int_{S_{B}} \phi^{0}\left(2 K \sin \beta \frac{\partial^{2} G^{0}}{\partial n \partial K}-2 i \frac{\partial}{\partial n} \frac{\partial^{2} G^{0}}{\partial y \partial K}\right) d S \\
& -2 i \int_{S_{F}} \phi^{0}\left(\nabla_{h} G^{0} \cdot \nabla_{h} \chi_{2}+\frac{1}{2} G^{0} \nabla_{h}^{2} \chi_{2}\right) d S=\left\{\begin{array}{cc}
-2 \pi \phi^{1 V}(\boldsymbol{x}) & \boldsymbol{x} \in S_{B} \\
-4 \pi \phi^{1 V}(\boldsymbol{x}) & \boldsymbol{x} \in \mathcal{V}
\end{array}\right.
\end{aligned}
$$

where the first case is an integral equation for $\phi^{1 V}$.

\section{The damping moment}

It is of principal interest to derive expressions for the wave drift damping force and moment. These quantities may be obtained in different ways. The most usual procedures are either by pressure integration over the wetted body surface, or by applying conservation of linear and angular momentum. Both methods have their advantages. Here we shall apply conservation of linear and angular momentum, giving as result compact formulae which are easy to evaluate numerically by a low-order boundary element method.

First we consider the time-averaged moment about the vertical axis, $M_{z}$. Conservation of angular momentum gives the following relation

$$
M_{z} \equiv \overline{\mathbf{k} \cdot \int_{S_{B}} p(\boldsymbol{x} \times \mathbf{n}) d S}=\overline{\mathbf{k} \cdot\left[-\rho \frac{d}{d t} \int_{V} \boldsymbol{x} \times \mathbf{v}^{\prime} d V-\int_{S(R)} p \boldsymbol{x} \times \mathbf{n} d S-\rho \int_{S(R)} \boldsymbol{x} \times \mathbf{v}^{\prime} \mathbf{v} \cdot \mathbf{n} d S\right]}
$$

As control surface a vertical circular cylinder $S(R)$ with axis passing through origo is applied, where we have that $\mathbf{k} \cdot(\boldsymbol{x} \times \mathbf{n})=0$ and $\mathbf{v} \cdot \mathbf{n}=\mathbf{v}^{\prime} \cdot \mathbf{n}$. Introducing $\mathbf{v}^{\prime}=\nabla \Phi^{\prime}$ we obtain

$$
M_{z}=\rho \Omega \frac{\partial}{\partial \beta} \overline{\int_{V} \mathbf{k} \cdot\left(\boldsymbol{x} \times \nabla \Phi^{\prime}\right) d V}-\rho \int_{S(R)} \overline{\Phi_{\theta}^{\prime} \Phi_{n}^{\prime}} d S
$$


where $\Phi_{\theta}^{\prime} \equiv \partial \Phi^{\prime} / \partial \theta, \Phi_{n}^{\prime} \equiv \partial \Phi^{\prime} / \partial n$. In obtaining $M_{z}$, terms being proportional to $\Omega^{2}, A^{3}$ are neglected. The integral in (68) over the fluid volume may be rewritten by applying Green's theorem, i.e.

$$
\overline{\mathbf{k} \cdot \int_{V} \boldsymbol{x} \times \nabla \Phi^{\prime} d V}=\overline{\int_{S_{B}+S_{F}} \mathbf{k} \cdot(\boldsymbol{x} \times \mathbf{n}) \Phi^{\prime} d S}=\int_{S_{B}} n_{6} \psi^{(2)} d S-\int_{S_{F}} \overline{\zeta_{\theta} \Phi^{\prime}} d S
$$

Here we have applied that $\mathbf{k} \cdot(\boldsymbol{x} \times \mathbf{n})=n_{6}$ at $S_{B}, \mathbf{k} \cdot(\boldsymbol{x} \times \mathbf{n})=-\zeta_{\theta}$ at $S_{F}, \mathbf{k} \cdot(\boldsymbol{x} \times \mathbf{n})=0$ at $S(R)$, and that $\overline{\Phi^{\prime}}=\psi^{(2)}$. By applying Green's theorem to $\chi_{6}$ and $\psi^{(2)}$ and exploiting the boundary conditions for $\chi_{6}$ and $\psi^{(2)}$, we may show that

$$
\int_{S_{B}} n_{6} \psi^{(2)} d S=\int_{S_{F}+S_{B}} \chi_{6} \psi_{n}^{(2)} d S=-\frac{A^{2} g}{2 \omega} \int_{S_{F}} \chi_{6} \operatorname{Im}\left(\phi^{0} \phi_{z z}^{0 *}\right) d S
$$

where we have applied (34) and that $\psi_{n}^{(2)}=0$ at $S_{B}$ (in the diffraction problem). Furthermore we have that

$$
\overline{\zeta_{\theta} \Phi^{\prime}}=\frac{A^{2} g}{2 \omega} \operatorname{Im}\left(\phi_{\theta} \phi^{*}\right), \quad \overline{\Phi_{\theta}^{\prime} \Phi_{n}^{\prime}}=\frac{A^{2} g}{2 K} \operatorname{Re}\left(\phi_{\theta} \phi_{n}^{*}\right)
$$

By then expanding the yaw-moment after $\epsilon$, i.e.

$$
M_{z}=M_{z 0}-\epsilon B_{66}
$$

where a minus sign is adopted since $-\epsilon B_{66}$ appears as a damping moment, and introducing $\phi=\phi^{0}+\epsilon \phi^{1}$, we obtain the following expression for $M_{z 0}$

$$
\frac{M_{z 0}}{\rho g A^{2}}=-\frac{1}{2 K} \int_{S(R)} R e\left[\phi_{\theta}^{0} \phi_{n}^{0 *}\right] d S
$$

This is a classical result, which agrees with Newman (1967), Grue and Palm (1993). For $B_{66}$ we find

$$
\frac{B_{66}}{\rho g A^{2}}=\frac{1}{2} \frac{\partial}{\partial \beta} \int_{S_{F}}\left[\chi_{6} \operatorname{Im}\left(\phi^{0} \phi_{z z}^{0 *}\right)+\operatorname{Im}\left(\phi_{\theta}^{0} \phi^{0 *}\right)\right] d S+\frac{1}{2 K} \int_{S(R)} \operatorname{Re}\left[\phi_{\theta}^{0} \phi_{n}^{1 *}+\phi_{\theta}^{1} \phi_{n}^{0 *}\right] d S
$$

We remark that all integrals in (74) are bounded for $R<\infty$. For $R \rightarrow \infty$, however, a part of the first integral and a part of the second integral both diverge. It will be shown below that these two parts cancel each other exactly.

The expression for $B_{66}$ may be brought into a form being more suitable for computations. First we rewite the integral over $S_{F}$ in (74). By applying Green's theorem to $\phi_{\beta}^{0 *}$ and $\phi^{1}$, and exploiting the body boundary conditions, i.e. $\phi_{\beta n}^{0 *}=\phi_{n}^{1}=0$ at $S_{B}$, we obtain

$$
\operatorname{Re} \int_{S_{F}}\left(\phi_{\beta}^{0 *} \phi_{n}^{1}-\phi^{1} \phi_{\beta n}^{0 *}\right) d S+R e \int_{S(R)}\left(\phi_{\beta}^{0 *} \phi_{n}^{1}-\phi^{1} \phi_{\beta n}^{0 *}\right) d S=0
$$

The integral over $S_{F}$ in (75) may be rewritten by introducing the free surface boundary conditions for $\phi_{\beta}^{0}$ and $\phi^{1}$, see (19) and (23), respectively, giving as result

$$
\begin{aligned}
R e \int_{S_{F}}\left(\phi_{\beta}^{0 *} \phi_{n}^{1}-\phi^{1} \phi_{\beta n}^{0 *}\right) & d S=-K \frac{\partial}{\partial \beta} \operatorname{Im} \int_{S_{F}}\left(\phi_{\theta}^{0} \phi^{0 *}-\phi^{0 *} \nabla_{h} \phi^{0} \cdot \nabla_{h} \chi_{6}\right) d S \\
- & K \operatorname{Im} \int_{S_{F}}\left(\frac{\partial}{\partial \theta}\left(\phi_{\beta}^{0 *} \phi^{0}\right)-\nabla_{h} \cdot\left(\phi_{\beta}^{0 *} \phi^{0} \nabla_{h} \chi_{6}\right)\right) d S
\end{aligned}
$$


It is easily shown by application of Gauss' theorem, exploiting that $\chi_{6 n}-(\mathbf{k} \times \boldsymbol{x}) \cdot \mathbf{n}=0$ at $C_{B}$ and $C(R), R \rightarrow \infty$, that the second integral on the right hand side of (76) equals zero. By then noting that $-\operatorname{Im}\left(\phi^{0 *} \nabla_{h} \phi^{0} \cdot \nabla_{h} \chi_{6}\right)=-\operatorname{Im} \nabla_{h} \cdot\left(\phi^{0 *} \chi_{6} \nabla_{h} \phi^{0}\right)+\chi_{6} \operatorname{Im}\left(\phi^{0} \phi_{z z}^{0 *}\right)$, and applying Gauss' theorem and the boundary conditions for $\phi^{0}$ and $\chi_{6}$, we may show that

$$
\frac{1}{2} \frac{\partial}{\partial \beta} \int_{S_{F}}\left[\chi_{6} \operatorname{Im}\left(\phi^{0} \phi_{z z}^{0 *}\right)+\operatorname{Im}\left(\phi_{\theta}^{0} \phi^{0 *}\right)\right] d S=-\frac{1}{2 K} R e \int_{S_{F}}\left(\phi_{\beta}^{0 *} \phi_{n}^{1}-\phi^{1} \phi_{\beta n}^{0 *}\right) d S
$$

By combining (74), (75) and (77) the expression for $B_{66}$ becomes

$$
\begin{aligned}
\frac{B_{66}}{\rho g A^{2}} & =\frac{1}{2 K} \operatorname{Re} \int_{S(R)}\left(\phi_{\beta}^{0 *} \phi_{n}^{1}-\phi^{1} \phi_{\beta n}^{0 *}\right) d S+\frac{1}{2 K} \int_{S(R)} \operatorname{Re}\left[\phi_{\theta}^{0} \phi_{n}^{1 *}+\phi_{\theta}^{1} \phi_{n}^{0 *}\right] d S \\
& =\frac{1}{2 K} \int_{S(R)} \operatorname{Re}\left[\left(\phi_{\beta}^{0 *}+\phi_{\theta}^{0 *}\right) \phi_{n}^{1}-\phi^{1 *}\left(\phi_{\beta}^{0}+\phi_{\theta}^{0}\right)_{n}\right] d S
\end{aligned}
$$

It is noted that the expression (78) for $B_{66}$ agrees with Newman (1993, eq. 5.10). The only difference between our and Newman's results is that in our analysis the rotation angle of the body is allowed to be of order one, as far as it is a slowly varying function of time, while in Newman's approach a perturbation expansion about a fixed position of the body is applied, assuming that the rotation angle and the angular velocity both are small. Thus, (78) being valid for a rotation angle of the body being not small, is a generalization of Newman (1993).

The expression for $B_{66}$ may be further developed by introducing $\phi^{1}=2 i K\left(\phi_{\beta K}^{0}+\phi_{\theta K}^{0}\right)+$ $\phi_{13}$, giving

$$
\frac{B_{66}}{\rho g A^{2}}=\frac{1}{2 K} R e \int_{S(R)}\left\{\left(\phi_{\beta}^{0 *}+\phi_{\theta}^{0 *}\right) \phi_{n}^{13}-\phi^{1 *}\left(\phi_{\beta}^{0}+\phi_{\theta}^{0}\right)_{n}+2 i K \frac{\partial}{\partial K}\left[\left(\phi_{\beta}^{0 *}+\phi_{\theta}^{0 *}\right)\left(\phi_{\beta}^{0}+\phi_{\theta}^{0}\right)_{n}\right]\right\} d S
$$

This integral may be evaluated by letting $R \rightarrow \infty$. $B_{66}$ may therefore be expressed in terms of the far-field forms (21) and (33) for $\phi_{7}$ and $\phi^{13}$, respectively, giving as final result

$$
\frac{B_{66}}{\rho g A^{2}}=\frac{1}{2 K} \operatorname{Im} \int_{0}^{2 \pi}\left[H_{\beta}^{0 *}(\theta)+H_{\theta}^{0 *}(\theta)\right] H^{1}(\theta) d \theta
$$

where the amplitude function $H^{1}$ is introduced by

$$
H^{1}=2 i K\left(H_{\beta K}^{0}+H_{\theta K}^{0}\right)+H^{13}
$$

$H^{0}$ and $H^{13}$ are given by (102) and (104), respectively. The formula (80) is very simple and is robust with respect to numerical evaluation of $B_{66}$.

\section{The damping force}

By applying conservation of linear momentum we obtain the following expression for the mean horizontal force, $\mathbf{F}=F_{1} \mathbf{i}_{1}+F_{2} \mathbf{i}_{2}$,

$$
F_{i}=\mathbf{i}_{i} \cdot\left[\rho \Omega \frac{\partial}{\partial \beta} \overline{\int_{V} \mathbf{v}^{\prime} d V}-\rho \Omega \times \overline{\int_{V} \mathbf{v}^{\prime} d V}-\overline{\int_{S(R)}\left(p \mathbf{n}+\rho \mathbf{v}^{\prime} \mathbf{v} \cdot \mathbf{n}\right) d S}\right], \quad i=1,2
$$


Using the same control surface as in the previous section, we may in the last term apply that $\mathbf{v} \cdot \mathbf{n}=\mathbf{v}^{\prime} \cdot \mathbf{n}$. In the derivations below, terms being proportional to $\Omega^{2}, A^{3}$ are neglected. Consider first the pressure-term in (82). By inserting (9) for the pressure we obtain

$$
\begin{aligned}
& \overline{\int_{S(R)}-\frac{p}{\rho} n_{i} d S}=\int_{C(R)} \overline{\left[\left(\Phi_{t}^{\prime}-\Omega \Phi_{\theta}^{\prime}\right) \zeta+\frac{1}{2} g \zeta^{2}\right]} n_{i} d l+\int_{S(R)}\left[\overline{\Phi_{t}^{\prime}}-\Omega \overline{\Phi_{\theta}^{\prime}}+\frac{1}{2} \overline{\left|\nabla \Phi^{\prime}\right|^{2}}\right] n_{i} d S \\
& =-\frac{1}{2 g} \int_{C(R)} \overline{\left[\Phi_{t}-\Omega \Phi_{\theta}\right]^{2}} n_{i} d l+\int_{S(R)}\left[-\Omega \psi_{\beta}^{(2)}-\Omega \psi_{\theta}^{(2)}+\frac{1}{2} \overline{|\nabla \Phi|^{2}}\right] n_{i} d S
\end{aligned}
$$

where we have exploited that the surface elevation far away from the body is given by $\zeta=-(1 / g)\left(\Phi_{t}-\Omega \Phi_{\theta}\right)$. By then introducing $\Phi=R e\left[(\right.$ Aig $\left./ \omega) \phi e^{i \omega t}\right]$, and noting that $\partial \Phi / \partial t=\operatorname{Re}\left[(A i g / \omega)\left(i \omega \phi-\Omega \phi_{\beta}\right) e^{i \omega t}\right]$, we obtain

$$
\begin{array}{r}
\overline{\int_{S(R)}-\frac{p}{\rho} n_{i} d S}=-\frac{g A^{2}}{4} \operatorname{Re} \int_{C(R)}\left[|\phi|^{2}-\frac{2 i \Omega}{\omega} \phi\left(\phi_{\beta}^{*}+\phi_{\theta}^{*}\right)\right] n_{i} d l \\
+\frac{g A^{2}}{4 K} \int_{S(R)}|\nabla \phi|^{2} n_{i} d S-\Omega \int_{S(R)}\left(\psi_{\beta}^{(2)}+\psi_{\theta}^{(2)}\right) n_{i} d S
\end{array}
$$

Consider then $\overline{\int_{V} \mathbf{v}^{\prime} d V}$. By using Gauss' theorem this integral may be rewritten as integrals over $S_{B}, S_{F}$ and $S(R)$. For the horizontal components we find

$$
\mathbf{i}_{i} \cdot \overline{\int_{V} \mathbf{v}^{\prime} d V}=\int_{S_{B}+S(R)} n_{i} \psi^{(2)} d S-\frac{A^{2} g}{2 \omega} \int_{S_{F}} \operatorname{Im}\left(\phi_{x_{i}} \phi^{*}\right) d S \quad i=1,2
$$

The integral over $S_{B}$ in (85) may be rewritten by applying Green's theorem to $\chi_{i}, i=1,2$, introduced in Section 3.1, and $\psi^{(2)}$, giving

$$
\int_{S_{B}} n_{i} \psi^{(2)} d S=\int_{S_{F}} \chi_{i} \psi_{n}^{(2)} d S=-\frac{A^{2} g}{2 \omega} \int_{S_{F}} \chi_{i} \operatorname{Im}\left(\phi \phi_{z z}^{*}\right) d S, \quad i=1,2
$$

where we have exploited the boundary conditions for $\psi^{(2)}$ and $\chi_{i}$. Next we apply Gauss' theorem to rewrite the integral over $S_{F}$ in (85), i.e.

$$
\begin{array}{r}
-\frac{A^{2} g}{2 \omega} \operatorname{Im} \int_{S_{F}} \nabla_{h} \phi \phi^{*} d S=-\frac{A^{2} g}{2 \omega} \operatorname{Im} \int_{S_{F}} \phi^{*} \nabla_{h} \boldsymbol{x}_{h} \cdot\left(\nabla_{h} \phi\right) d S \\
=\frac{A^{2} g}{2 \omega} \operatorname{Im}\left[\int_{S_{F}} \boldsymbol{x}_{h} \phi \phi_{z z}^{*} d S+\int_{C_{B}+C(R)} \boldsymbol{x}_{h} \phi \phi_{n}^{*} d S\right]
\end{array}
$$

where we have introduced $\boldsymbol{x}_{h}=x \mathbf{i}+y \mathbf{j}$. In the diffraction problem $\partial \phi / \partial n=0$ at $C_{B}$, provided that the body is wallsided. By inserting (84) - (88) into (82) we find for the force

$$
\begin{aligned}
\frac{\mathbf{F}}{\rho g A^{2}}= & \frac{\epsilon}{2}\left(-\mathbf{i}_{i} \frac{\partial}{\partial \beta}+\mathbf{k} \times \mathbf{i}_{i}\right)\left[\int_{S_{F}}\left(\chi_{i}-x_{i}\right) \operatorname{Im}\left(\phi \phi_{z z}^{*}\right) d S-\int_{C(R)} x_{i} \operatorname{Im}\left(\phi \phi_{n}^{*}\right) d l\right] \\
+ & \frac{\mathbf{i}_{i}}{4 K} \operatorname{Re}\left[-K \int_{C(R)} \phi \phi^{*} n_{i} d l+\int_{S(R)}\left[\nabla \phi \cdot \nabla \phi^{*} n_{i}-2 \phi_{x_{i}} \phi_{n}^{*}\right] d S\right] \\
& -\frac{\epsilon \mathbf{i}_{i}}{2} \int_{C(R)} \operatorname{Im}\left[\phi\left(\phi_{\beta}^{*}+\phi_{\theta}^{*}\right)\right] n_{i} d l
\end{aligned}
$$


where the sum is over $i=1,2$. By applying a variety of Stokes' theorem we may show that, see eq. (114) in Appendix B,

$$
-\int_{C} K \phi \phi^{*} n_{i} d l+\int_{S}\left[\nabla \phi \cdot \nabla \phi^{*} n_{i}-\phi_{x_{i}} \phi_{n}^{*}\right] d S=\int_{S} \phi\left(\phi_{x_{i}}^{*}\right)_{n} d S-2 i K \epsilon \int_{C} \phi\left(\phi_{\beta}^{*}+\phi_{\theta}^{*}\right) n_{i} d l
$$

By combining (89) and (90) we find

$$
\begin{aligned}
\frac{\mathbf{F}}{\rho g A^{2}}=\frac{\epsilon}{2} & \left(-\mathbf{i}_{i} \frac{\partial}{\partial \beta}+\mathbf{k} \times \mathbf{i}_{i}\right)\left[\int_{S_{F}}\left(\chi_{i}-x_{i}\right) \operatorname{Im}\left(\phi \phi_{z z}^{*}\right) d S-\int_{C(R)} x_{i} \operatorname{Im}\left(\phi \phi_{n}^{*}\right) d l\right] \\
& +\frac{\mathbf{i}_{i}}{2 K} \operatorname{Re} \int_{S(R)}\left[\phi\left(\phi_{x_{i}}^{*}\right)_{n}-\phi_{x_{i}} \phi_{n}^{*}\right] d S
\end{aligned}
$$

By then introducing $\phi=\phi^{0}+\epsilon \phi^{1}$, and expanding the force after $\epsilon$, i.e. $\mathbf{F}=\mathbf{F}_{0}-\epsilon\left(B_{16} \mathbf{i}+\right.$ $\left.B_{26} \mathbf{j}\right)$, where a minus sign is adopted since $-\epsilon\left(B_{16} \mathbf{i}+B_{26} \mathbf{j}\right)$ formally appears as a damping force, we find

$$
\begin{gathered}
\frac{\mathbf{F}_{0}}{\rho g A^{2}}=\frac{1}{4 K} \operatorname{Re} \int_{S(R)}\left[\phi^{0}\left(\nabla_{h} \phi^{0 *}\right)_{n}-\phi_{x_{i}}^{0} \phi_{n}^{0 *}\right] d S \\
\frac{B_{16} \mathbf{i}+B_{26} \mathbf{j}}{\rho g A^{2}}=\operatorname{Im} \frac{1}{2}\left(\mathbf{i}_{i} \frac{\partial}{\partial \beta}-\mathbf{k} \times \mathbf{i}_{i}\right)\left[\int_{S_{F}}\left(\chi_{i}-x_{i}\right) \phi^{0} \phi_{z z}^{0 *} d S-\int_{C(R)} x_{i} \phi^{0} \phi_{n}^{0 *} d l\right] \\
-\operatorname{Re} \frac{1}{4 K} \int_{S(R)}\left[\phi^{0}\left(\nabla_{h} \phi^{1 *}\right)_{n}+\phi^{1}\left(\nabla_{h} \phi^{0 *}\right)_{n}-\phi_{n}^{0} \nabla_{h} \phi^{1 *}-\phi_{n}^{1} \nabla_{h} \phi^{0 *}\right] d S
\end{gathered}
$$

Evaluation of the integrals in (93) requires some algebra, which is outlined in Appendix C. It is found that $B_{16}$ and $B_{26}$ may be expressed in terms of the far-field amplitudes of the potentials $\phi^{7}$ and $\phi^{13}$ as well as an integral over the free surface, giving as result

$$
\begin{aligned}
& \frac{B_{16} \mathbf{i}+B_{26} \mathbf{j}}{\rho g A^{2}}=\frac{1}{2}\left(\mathbf{i}_{i} \frac{\partial}{\partial \beta}-\mathbf{k} \times \mathbf{i}_{i}\right) \int_{S_{F}}\left(\chi_{i}-x_{i}\right) \operatorname{Im}\left(\phi^{0} \phi_{z z}^{0 *}\right) d S \\
& +\frac{1}{2} \operatorname{Re} \int_{0}^{2 \pi}\left[H^{0}(\theta) H^{1 *}(\theta)\left(\begin{array}{c}
\cos \theta \\
\sin \theta
\end{array}\right)-2 i H^{0}(\theta)\left(H_{\beta \theta}^{0 *}(\theta)+H_{\theta \theta}^{0 *}(\theta)\right)\left(\begin{array}{c}
-\sin \theta \\
\cos \theta
\end{array}\right)\right] d \theta \\
& +\frac{1}{2} \operatorname{Re}\left\{\sqrt{\frac{2 \pi}{K}} e^{i \pi / 4}\left[H^{1 *}(\beta)\left(\begin{array}{c}
\cos \beta \\
\sin \beta
\end{array}\right)-2 i\left(H_{\beta \theta}^{0 *}(\beta)+H_{\theta \theta}^{0 *}(\beta)\right)\left(\begin{array}{c}
-\sin \beta \\
\cos \beta
\end{array}\right)\right]\right\}
\end{aligned}
$$

We note that $\int_{S_{F}}\left(\chi_{i}-x_{i}\right) \operatorname{Im}\left(\phi^{0} \phi_{z z}^{0 *}\right) d S, i=1,2$, denote the horizontal dipole moments of the potential $\psi^{(2)}$ for $R \rightarrow \infty$. These integrals have relatively quick convergence, since $\operatorname{Im}\left(\phi^{0} \phi_{z z}^{0 *}\right)$ quite rapidly tends to zero with increasing distance from the body, see Grue and Palm (1993). 


\section{Conservation of energy}

In the general case the energy equation reads

$$
\frac{W}{\rho}=-\overline{\frac{d}{d t} \int_{V}\left(\frac{1}{2} \mathbf{v}^{2}+g z\right) d V}-\overline{\int_{S(R)}\left(\frac{p}{\rho}+\frac{1}{2} \mathbf{v}^{2}+g z\right) \mathbf{v} \cdot \mathbf{n} d S}
$$

where $W=\overline{\int_{S_{B}} p \mathbf{v} \cdot \mathbf{n} d S}$ denotes the mean work performed by the pressure-force on the body. In the present example the body is restrained, and no work is performed, thus $W=0$. Equation (95) may then be utilized as a check on the model and the computational procedure.

As in the previous sections, $\mathbf{v} \cdot \mathbf{n}=\mathbf{v}^{\prime} \cdot \mathbf{n}$ at $S(R)$. Furthermore we have $-\overline{\frac{d}{d t} \int_{V}\left(\frac{1}{2} \mathbf{v}^{2}+g z\right) d V}=\Omega \frac{\partial}{\partial \beta} \overline{\int_{V}\left(\frac{1}{2} \mathbf{v}^{2}+g z\right) d V}$. It may be shown that

$$
\overline{\int_{V}\left(\frac{1}{2} \mathbf{v}^{2}+g z\right) d V}=\frac{A^{2} g}{2} \int_{S_{F}}\left|\phi^{0}\right|^{2} d S+\frac{A^{2} g}{4 K} R e \int_{S(R)} \phi^{0} \phi_{n}^{0 *} d S
$$

where we have neglected terms proportional to $\Omega$. Furthermore we have

$$
-\overline{\int_{S(R)}\left(\frac{p}{\rho}+\frac{1}{2} \mathbf{v}^{2}+g z\right) \mathbf{v}^{\prime} \cdot \mathbf{n} d S}=\int_{S(R)} \overline{\Phi_{t}^{\prime} \Phi_{n}^{\prime}} d S=\frac{A^{2} g^{2}}{2 \omega^{2}} R e \int_{S(R)}\left(i \omega \phi-\Omega \phi_{\beta}\right) \phi_{n}^{*} d S
$$

By introducing $\phi=\phi^{0}+\epsilon \phi^{1}$ and expanding the right hand side of (95) by $W^{0}+\epsilon W^{1}$, we obtain

$$
\begin{gathered}
\frac{W^{0}}{\rho g A^{2} c_{g}}=-\operatorname{Im} \int_{S(R)} \phi^{0} \phi_{n}^{0 *} d S \\
\frac{W^{1}}{\rho g A^{2} c_{g}}=\frac{\partial}{\partial \beta}\left[K \int_{S_{F}}\left|\phi^{0}\right|^{2} d S+R e \frac{1}{2} \int_{S(R)} \phi^{0} \phi_{n}^{0 *} d S\right] \\
-I m \int_{S(R)}\left(\phi^{1} \phi_{n}^{0 *}+\phi^{0} \phi_{n}^{1 *}+i \phi_{\beta}^{0} \phi_{n}^{0 *}\right) d S
\end{gathered}
$$

where we have introduced the group velocity of the waves, $c_{g} \equiv \partial \omega / \partial K=(g / 2 \omega)$. By then applying $\phi^{1}=\phi^{11}+\phi^{12}+\phi^{13}$, we may after some algebra obtain that

$$
\frac{W^{1}}{\rho g A^{2} c_{g}}=-2 K \frac{\partial}{\partial K} \operatorname{Re} \int_{S(R)} \phi_{\theta}^{0} \phi_{n}^{0 *} d S-\operatorname{Im} \int_{S(R)}\left(\phi^{13} \phi_{n}^{0 *}+\phi^{0} \phi_{n}^{13 *}\right) d S
$$

Inserting for $\phi^{0}$ and $\phi^{13}$, and letting $R \rightarrow \infty$, we obtain

$$
\begin{aligned}
\frac{W^{1}}{\rho g A^{2} c_{g}}= & -K \frac{\partial}{\partial K} I m\left[\int_{0}^{2 \pi} H^{0}(\theta) H_{\theta}^{0}(\theta)^{*} d \theta+\sqrt{\frac{8 \pi}{K}} H_{\theta}^{0}(\beta)^{*} e^{i \pi / 4}\right] \\
& -\operatorname{Re}\left[\int_{0}^{2 \pi} H^{0}(\theta) H^{13}(\theta)^{*} d \theta+\sqrt{\frac{2 \pi}{K}} H^{13}(\beta)^{*} e^{i \pi / 4}\right]
\end{aligned}
$$

where the method of stationary phase is applied to obtain the terms containing $H_{\theta}^{0}(\beta)$ and $H^{13}(\beta)$. 


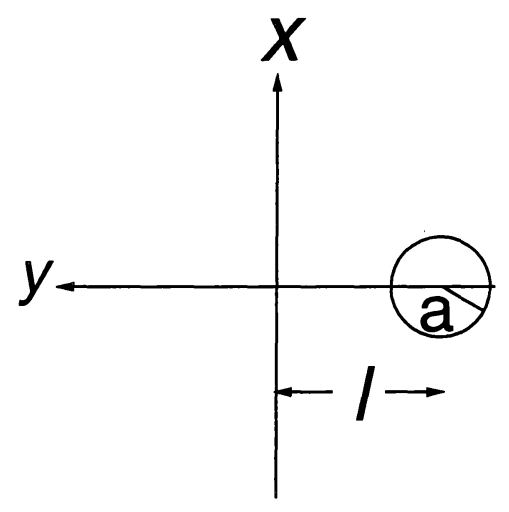

Figure 1: Sketch of the location of a vertical circular cylinder with radius $a$ and draught $3 a$. The axis is located at $x=0, y=-l$. The cylinder and the free surface are both discretized with 784 panels. $S_{F}$ is discretized out to the circle with center in the cylinder axis and radius $7 a$.

\section{Numerical implementation and results}

We consider then the numerical implementation of the theory outlined above and some numerical results for the damping coefficients $B_{i 6}, i=1,2,6$, for practical geometries. The first step is to evaluate the various velocity potentials. More specifically, the potentials $\phi^{0}, \phi_{\beta}^{0}, \phi_{K}^{0}, \phi_{\beta K}^{0}$, and $\phi^{1}-\phi^{11}$ are required at the body surface $S_{B}$, and the potentials $\phi^{0}$, $\phi_{\beta}^{0}, \phi_{K}^{0}, \chi_{1}, \chi_{2}, \chi_{6}$, at $S_{F}$. $\phi^{0}$ is obtained by (38). By differentiating (38) with respect to either $\beta$ or $K$, integral equations are obtained for $\phi_{\beta}^{0}$ and $\phi_{K}^{0}$, respectively. Then $\phi_{\beta K}^{0}$ is found from (41), and $\phi^{1}-\phi^{11}$ from (50). The potentials $\chi_{i}, i=1,2,6$, are obtained by source distributions.

The set of potentials and source distributions are solved by means of their respective integral equations, applying a low-order panel method as numerical method. The body surface and the free surface are then discretized by quadrilaterals, and the potential or source strength is taken as a constant at each panel. The Green function $G^{0}$ and its derivatives involved in the integral equations have singularities $\nabla 1 / r, \nabla 1 / r^{\prime}, 1 / r, 1 / r^{\prime}$, where $r=\left[(x-a)^{2}+(y-b)^{2}+(z-c)^{2}\right]^{1 / 2}$ and $r^{\prime}=\left[(x-a)^{2}+(y-b)^{2}+(z+c)^{2}\right]^{1 / 2}$. These singularities are integrated separately over each panel by analytical methods, see Newman (1985). Otherwise a midpoint rule is applied for numerical integration.

The formulae for $B_{i 6}$ require that the far-field amplitudes $H^{0}$ and $H^{13}$ of $\phi_{7}$ and $\phi^{13}$, respectively, are determined. By introducing the far-field form of $G^{0}$ into (38), we obtain for $H^{0}$

$$
H^{0}=-\frac{1}{4 \pi} \int_{S_{B}} \phi^{0} \frac{\partial h^{0}}{\partial n} d S
$$

where $h^{0}$ is given by (37). Derivatives of $H^{0}$ with respect to $\beta, \theta, K$, or combinations of these variables, are easily obtained. $H_{\beta}^{0}$ is e.g. found by $H_{\beta}^{0}=-1 /(4 \pi) \int_{S_{B}} \phi_{\beta}^{0} h_{n}^{0} d S$. Consider then $\phi^{13}$, which is obtained by subtracting $\phi^{12}$ from $\phi^{1}-\phi^{11}$. $\phi^{12}$ may be obtained by differentiating (38) with respect to $\theta$ and $K$ and multiplying by $2 i K$. By 


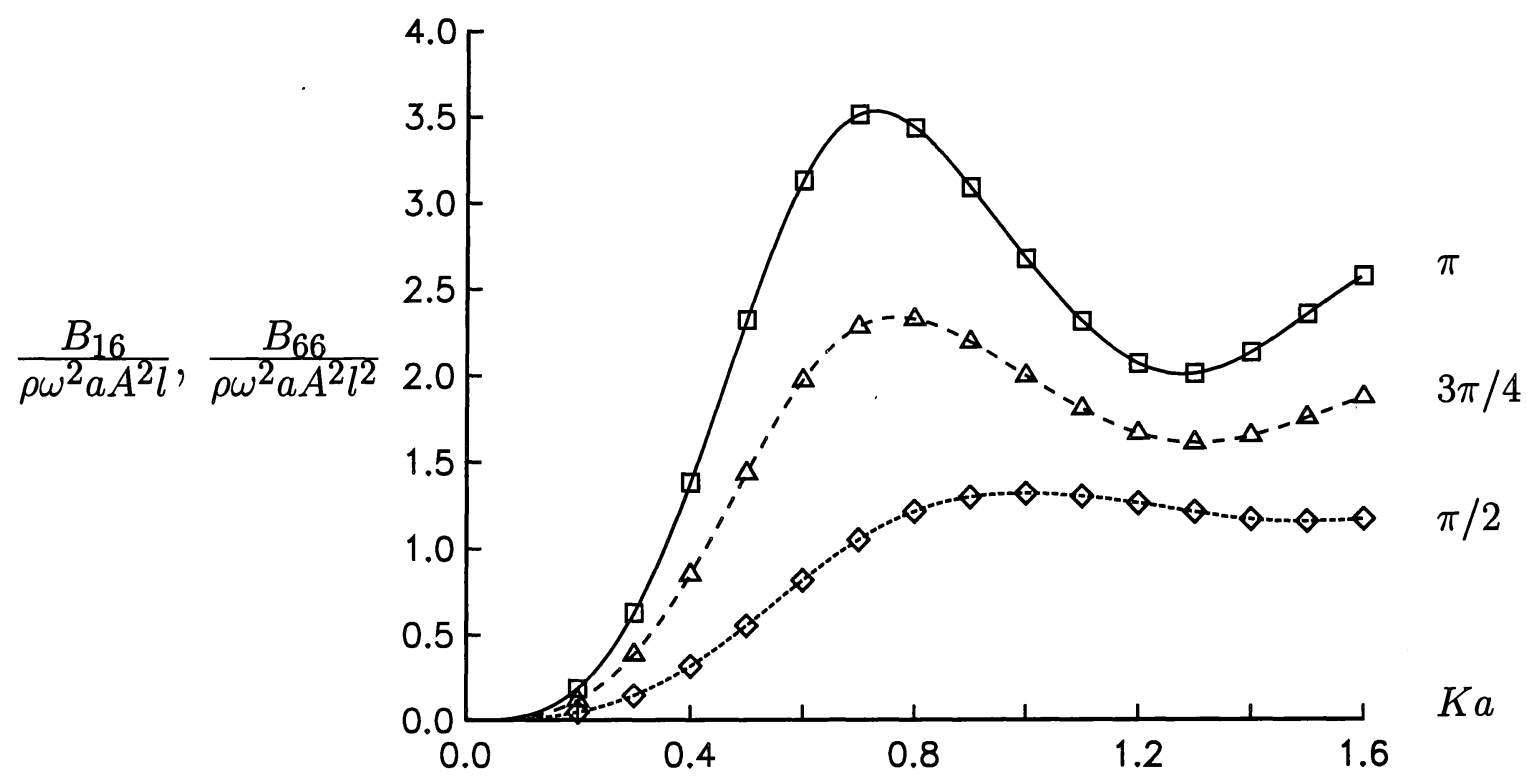

Figure 2: Numerical results for $B_{16} / \rho \omega^{2} a A^{2} l$ and $B_{66} / \rho \omega^{2} a A^{2} l^{2}$ vs. $K a$ for the vertical cylinder described in figure 1 . Three different wave headings. Wave amplitude $A$, wave number $K$. $B_{16} / \rho g A^{2} l$ : Solid line, $\beta=\pi$. Dashed line, $\beta=3 \pi / 4$. Dotted line, $\beta=\pi / 2$. $B_{66} / \rho \omega^{2} a A^{2} l^{2}$ : Squares, $\beta=\pi$. Triangles, $\beta=3 \pi / 4$. Diamonds, $\beta=\pi / 2$.

subtracting the result from (50) we then obtain for $\phi^{13}$

$$
\begin{aligned}
4 \pi \phi^{13}(\boldsymbol{x})= & \int_{S_{B}}\left(\phi^{11}-\phi^{1}\right) \frac{\partial G^{0}}{\partial n} d S+2 i K \int_{S_{B}} \phi_{K}^{0} \frac{\partial^{2} G^{0}}{\partial n \partial \theta} d S \\
& +2 i K \int_{S_{F}} \phi^{0}\left(\nabla_{h} \chi_{6} \cdot \nabla_{h} G^{0}+\frac{1}{2} G^{0} \nabla_{h}^{2} \chi_{6}\right) d S
\end{aligned}
$$

for $\boldsymbol{x} \in \mathcal{V}$. By then introducing the far-field form of $G^{0}$ we obtain

$$
\begin{aligned}
H^{13} & =-\frac{1}{4 \pi} \int_{S_{B}}\left(\phi^{1}-\phi^{11}\right) \frac{\partial h^{0}}{\partial n} d S+\frac{i K}{2 \pi} \int_{S_{B}} \phi_{K}^{0} \frac{\partial}{\partial n} \frac{\partial h^{0}}{\partial \theta} d S \\
& +\frac{i K}{2 \pi} \int_{S_{F}} \phi^{0}\left(\nabla_{h} \chi_{6} \cdot \nabla_{h} h^{0}+\frac{1}{2} h^{0} \nabla_{h}^{2} \chi_{6}\right) d S
\end{aligned}
$$

with $h^{0}$ is given by (37).

Results for $B_{i 6}, i=1,2,6$, are then considered for two different geometries. In the first example the body is a vertical circular cylinder with radius $a$, draught $3 a$, and located with its axis at $x=0, y=-l$, where $l$ is arbitrary, see figure 1 . In the absolute frame of reference the cylinder-axis describes a circular path about the origin with radius $l$. Since the body is a vertical circular cylinder, the moment with respect to the $z$-axis equals the force-component along the $x$-axis multiplied by the arm $l$, i.e. $M_{z 0}=l \mathbf{F}_{0} \cdot \mathbf{i}, B_{66}=l B_{16}$. In figure 2 are shown results for the damping coefficients $B_{16}$ normalized by $\rho \omega^{2} a A^{2} l$ and $B_{66}$ normalized by $\rho \omega^{2} a A^{2} l^{2}$ for three different wave angles. The numerical results confirm that $B_{16} / l$ and $B_{66} / l^{2}$ are independent of the value of $l$, and that $B_{66}=l B_{16}$, which both 


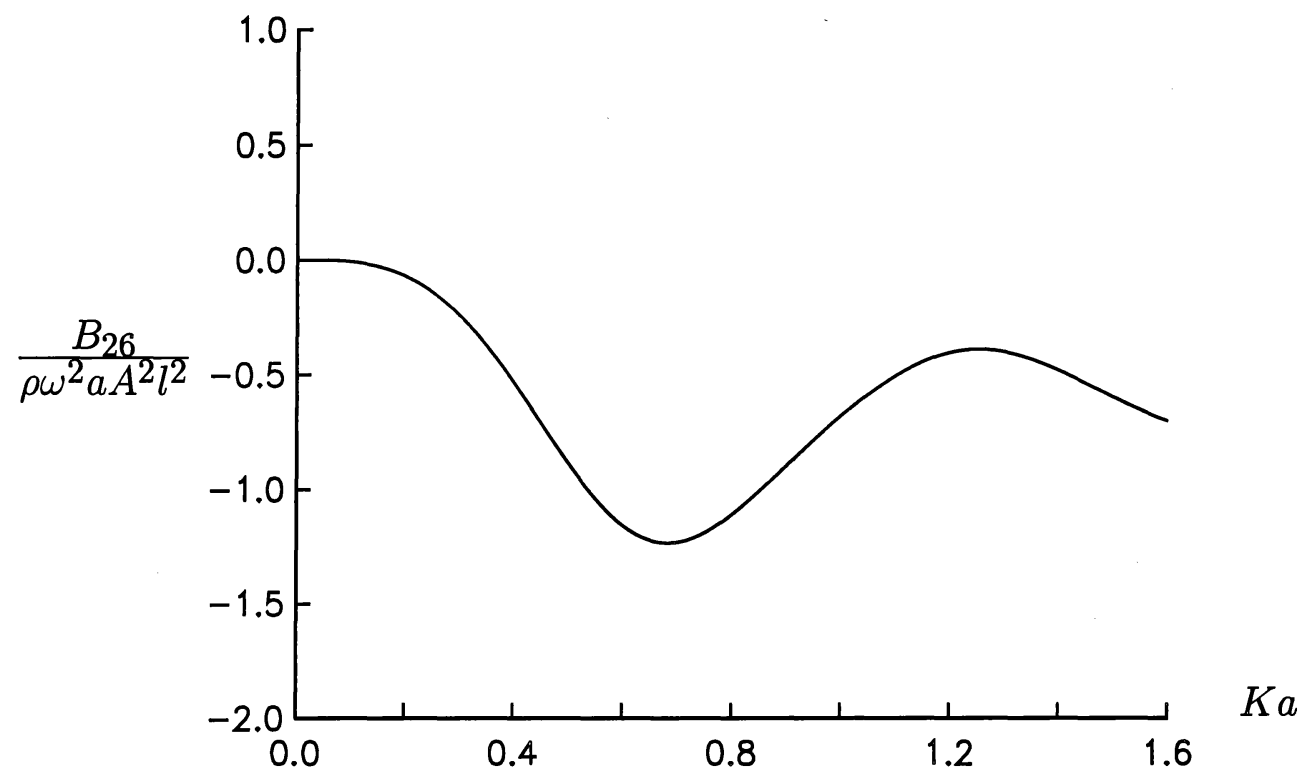

Figure 3: Numerical results for $B_{26} / \rho \omega^{2} a A^{2} l$ vs. $K a$ for the vertical cylinder described in figure 1. $\beta=3 \pi / 4$. $B_{26}=0$ for $\beta=\pi / 2$ and $\beta=\pi$.

are expected results. We remark that the variation of the damping coefficients $B_{16}$ and $B_{66}$ with respect to the wave angle, keeping the wave number fixed, satisfy the following relations for this example: $B_{16}(\pi / 2) \leq B_{16}(\beta) \leq B_{16}(\pi)$ and $B_{66}(\pi / 2) \leq B_{66}(\beta) \leq B_{66}(\pi)$ for all $\beta$.

In figure 3 is shown $B_{26}$ for wave angle $\beta=3 \pi / 4 . \quad B_{26}$ is always negative or zero. The numerical results confirm, as expected, that $B_{26} / l$ is independent of the value of $l$. For this geometry, it is noted that $B_{16} / l, B_{26} / l$, and $B_{66} / l^{2}$ may be obtained from the wave drift damping coefficients due to a vertical cylinder translating along the positive $x$-direction. Indeed, the results shown in figures 2 and 3 are in exellent agreement with the corresponding wave drift damping coefficients using the method of Nossen, Grue and Palm (1991) for the translatory case.

We have also invoked the energy equation for the vertical cylinder, see eqs. (95), (98), (101), which in our case shall predict that $W=0$, since the body performs no work on the fluid. In all computations we find that $W^{0} \simeq 0$ and $W^{1} \simeq 0$. For example, in computing $W^{1}$, we find that both terms on the r.h.s. of (101) are large, but cancel each other almost exactly, see figure 4 .

In the next example the geometry is a ship, length $l$ and beam $b_{0}$, with $l / b_{0}=5.6$. The ship section is a half circular cylinder with radius $r(x)=0.5 b_{0}\left[1-(2 x / l)^{4}\right],|x| \leq l / 2 . S_{B}$ and $S_{F}$ are both discretized with 800 panels. $S_{F}$ is discretized out to a circle with radius $l$ and centre in the origin. Numerical values of $B_{66}$ are shown in figure 5 for $\beta=\pi / 2$ (beam seas), $\beta=3 \pi / 4$ (quartering seas), $\beta=\pi$ (head seas). $B_{66}$ is always positive, is largest for beam seas, and smallest for head seas. More specifically, $B_{66}$ is $4-5$ times larger for beam seas than for head seas, when $K l>6$.

In figures 6 and 7 are displayed results for $B_{16}$ and $B_{26}$ for the ship. $B_{16}$ and $B_{26}$ attain 


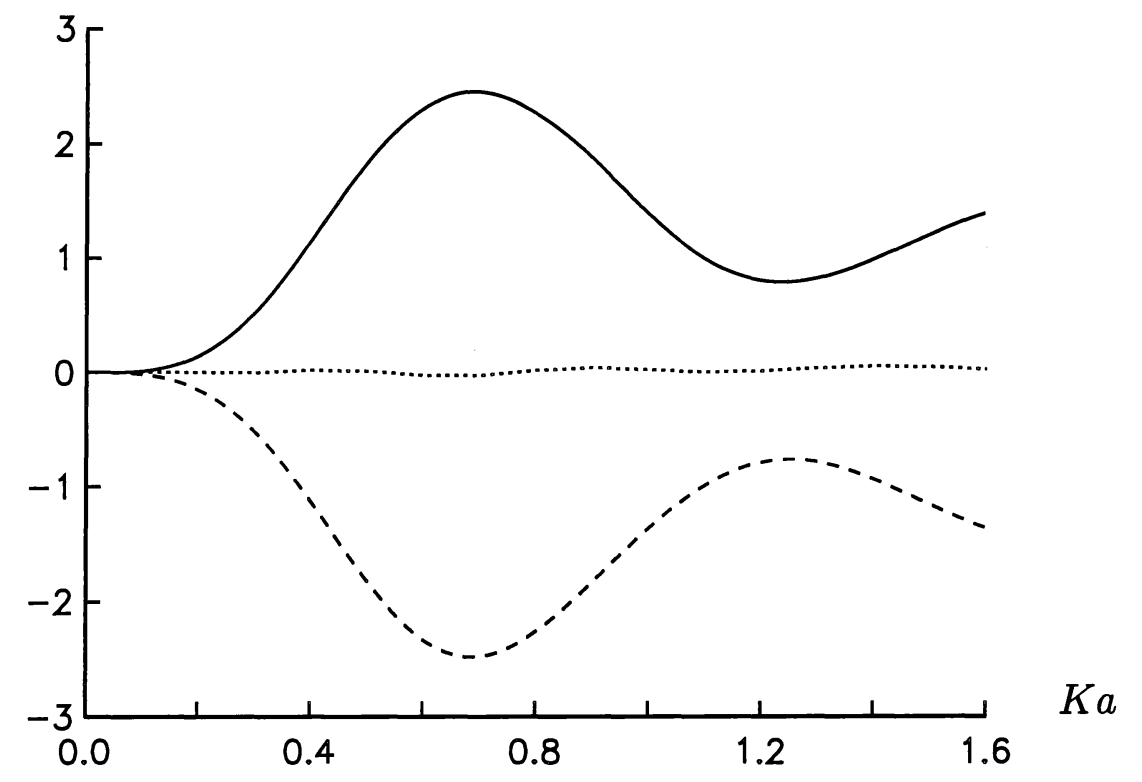

Figure 4: Energy equation vs. $K a$ for the vertical cylinder described in figure 1. $\beta=\pi$. Solid line: The first term on the r.h.s. of eq. (101) divided by $l$. Dashed line: The second term on the r.h.s. of eq. (101) divided by $l$. Dotted line: $W^{1} / \rho g A^{2} c_{g} l$.

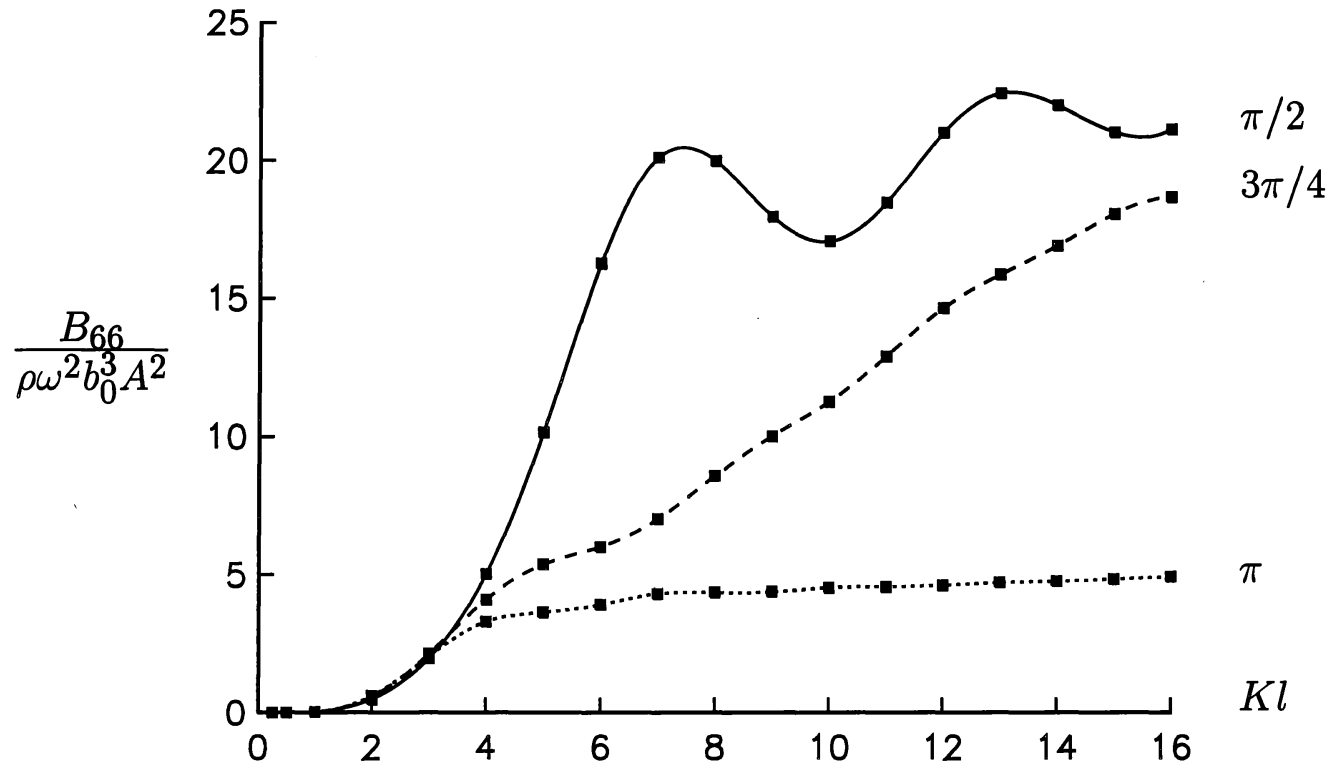

Figure 5: $B_{66} / \rho \omega^{2} b_{0}^{3} A^{2}$. vs. $K l$ for the ship. Solid line: $\beta=\pi / 2$. Dashed line: $\beta=3 \pi / 4$. Dotted line: $\beta=\pi$. The black squares denote the computation points. 


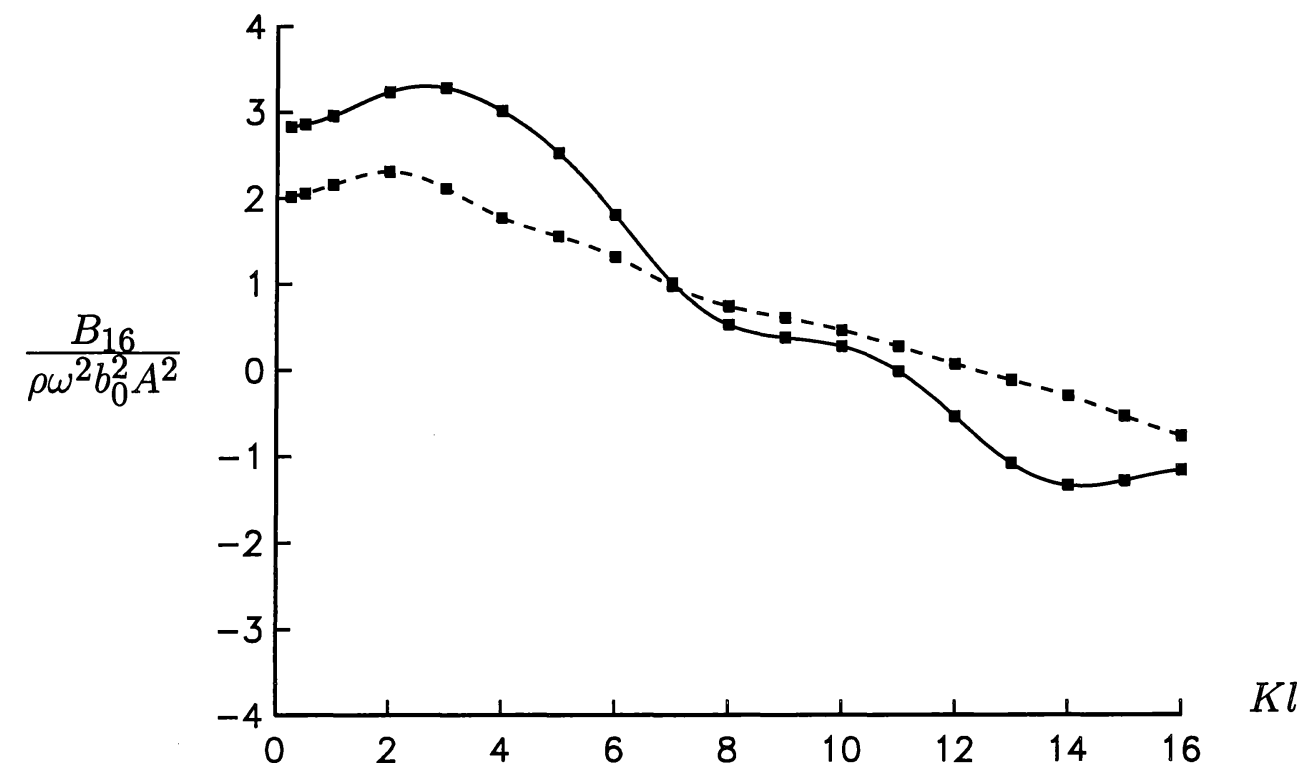

Figure 6: $B_{16} / \rho \omega^{2} b_{0}^{2} A^{2}$ vs. $K l$ for the ship. Solid line: $\beta=\pi / 2$. Dashed line: $\beta=3 \pi / 4$. $B_{16}=0$ for $\beta=\pi$.

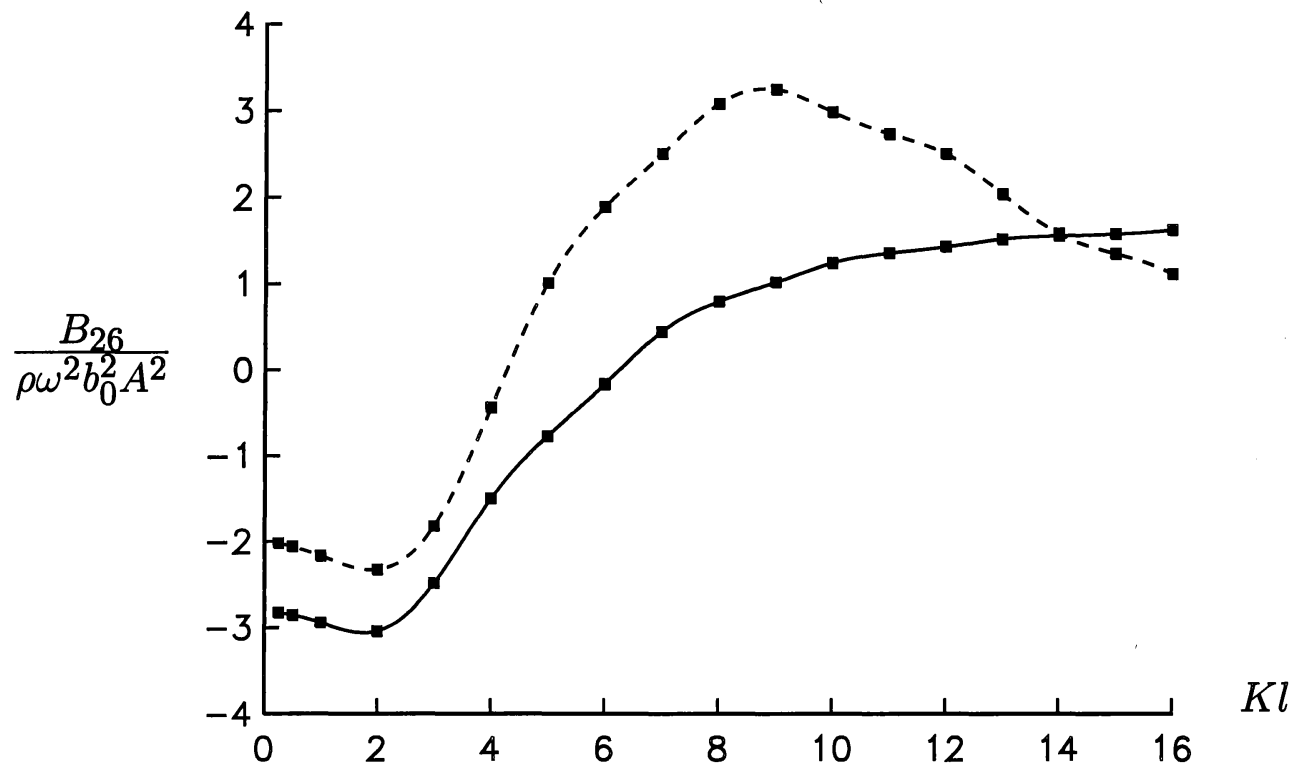

Figure 7: $B_{26} / \rho \omega^{2} b_{0}^{2} A^{2}$ vs. $K l$ for the ship. Solid line: $\beta=\pi$. Dashed line: $\beta=3 \pi / 4$. $B_{26}=0$ for $\beta=\pi / 2$. 


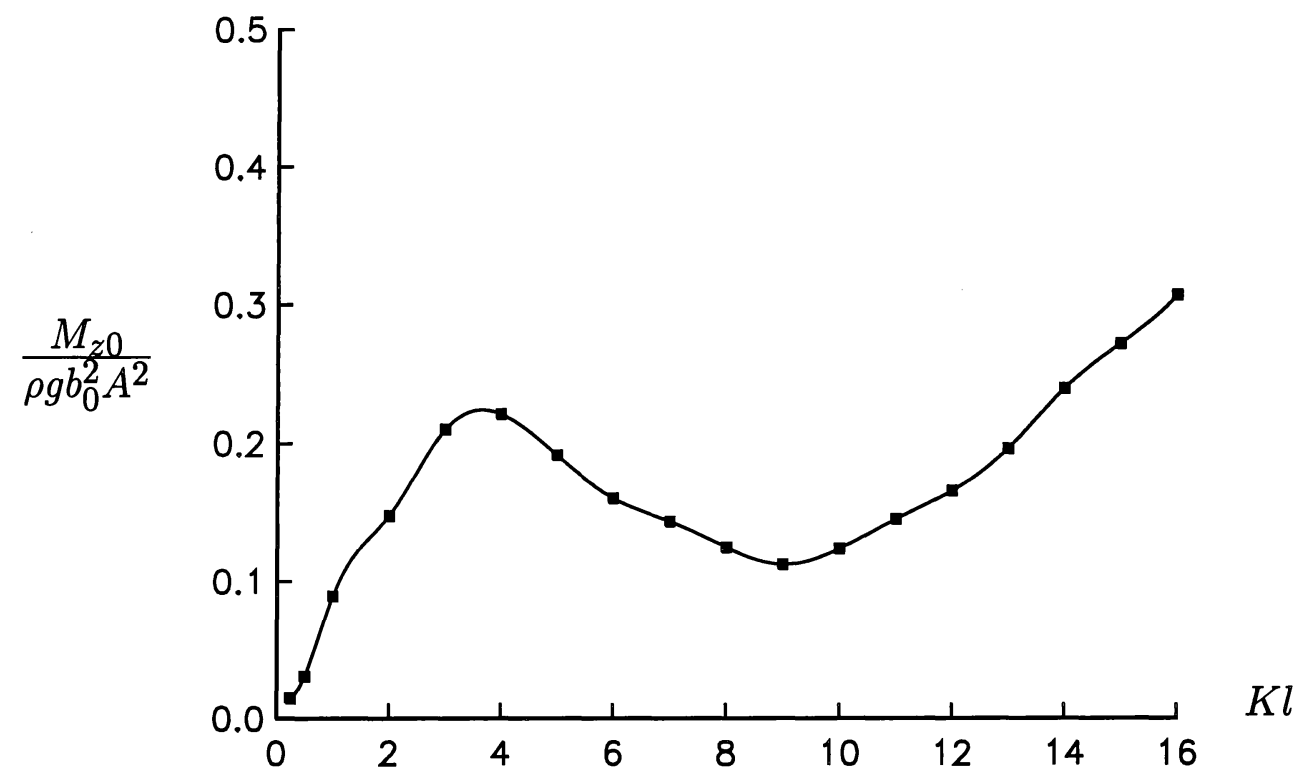

Figure 8: $M_{z 0} / \rho g b_{0}^{2} A^{2}$ vs. $K l$ for the ship. $\beta=3 \pi / 4$,

both positive and negative values. For long waves we find that $B_{16}(\pi / 2)=-B_{26}(\pi)$, $B_{16}(\beta)=B_{16}(\pi / 2) \sin \beta$, and $B_{26}(\beta)=B_{26}(\pi) \cos (\pi-\beta)$. It is noted that $B_{16}$ for beam seas and $B_{26}$ for head seas both are large. These results correspond to nonvanishing moments with respect to the $z$-axis, due to a ship translating respectively along the $x$ axis in beam seas, and along the $y$-axis in head seas, see Grue and Palm (1993).

It is of interest to compare the magnitude of $B_{66}$ to the zero speed moment $M_{z 0}$, where the latter is displayed in figure 8 for $\beta=3 \pi / 4$. We observe that $B_{66}$ is one order of magnitude larger that $M_{z 0}$. For example, for $\beta=3 \pi / 4$ and $K l=10$, we have $B_{66} / M_{z 0} \simeq 200$. Thus, if the slow angular velocity $\Omega$ is $5 \%$ of the wave frequency $\omega$, the value of $(\Omega / \omega) B_{66}$ is still 10 times larger than $M_{z 0}$ in this example. This means that $B_{66}$ provides a considerable damping moment for a slow rotational motion of a moored ship.

\section{Concluding remarks}

A method for evaluating the diffracted waves, the wave forces and the wave drift damping due to a floating body performing a slow rotation about the vertical axis, with a finite rotation angle, is developed. The incoming waves, with wave frequency $\omega$ in the absolute frame of reference, and the slow angular velocity $\Omega$ of the body, where it is assumed that $\Omega<<\omega$, introduce two time scales to the problem, being proportional to $1 / \omega$ and $1 / \Omega$, respectively. It is noted that the components of the exciting force experienced by the body, being proportional to the wave amplitude, generally will oscillate with a frequency $\omega+O(\Omega)$, being slightly different from $\omega$ and varying with respect to the wave angle. The corresponding result is true for the linear fluid flow at a fixed space location.

The mathematical problem is formulated by means of potential theory. A set of boundary value problems is developed by applying perturbation expansions after the incoming wave amplitude and the slow angular velocity of the body, which are solved by means of integral 
equations, containing unknown quantities on the wetted body surface only. It is noted that the boundary value problems and the corresponding integral equations due to a slowly translating body are obtained as part-results of the present analysis.

Formulae for the wave drift damping coefficients are obtained by applying conservation of linear and angular momentum. The resulting expression for the damping coefficient $B_{66}$ given by eq. (78), agrees with Newman (1993, eq. 5.10). The only difference between our and Newman's formula for $B_{66}$ is that in the present method the rotation angle of the body is allowed to be of arbitrary magnitude, while in Newmans's analysis the rotation angle is assumed small. Our theory thus shows that the magnitude of the rotation angle is irrelevant to the problem, provided that the angular velocity of the body is small compared to the wave frequency. The expressions for the damping coefficients $B_{16}, B_{26}$, given by eq. (93), bear strong similarities with the corresponding formulae derived by Newman (1993, eq. 5.6). The mathematical expressions are not identical, however. Here we proceed one step further than Newman (1993), as the wave drift damping coefficients are expressed in terms of the far-field amplitudes of the wave potentials involved, resulting in simple formulae being suitable for efficient numerical algoritms. Numerical solution of the problem is obtained by using a low-order panel method. The method is applicable to geometries of general form.

Examples for the damping coefficients $B_{16}, B_{26}, B_{66}$, are considered for two different bodies, viz. a symmetric ship and a vertical circular cylinder with axis describing a circular path in the horizontal plane. Results for other geometries may be found in Grue (1994). The method is carefully checked e.g. by invoking the balance of energy, which in all examples is satisfied to a relative accuracy being better than $1 \%$. We find that the damping coefficients are one order of magnitude larger than the time-averaged horizontal force components $F_{x 0}, F_{y 0}$, and the vertical moment $M_{z 0}$, which means that a slow rotation of the body introduces a significant change of the forces. The damping coefficient $B_{66}$ is in all present examples found to be positive and large. This means that wave drift damping due to a slow yaw-motion of the body is just as pronounced as for slow translatory motions. Thus, evaluation of the complete wave drift damping matrix eq. (2) is required to realistically study slow drift motions of moored floating bodies in the three horizontal modes.

In the present contribution only the linear diffraction effects are taken into account, which means that the body is kept fixed in the relative frame of reference. The method may, however, be generalized to account for the linear responses of the floating body and the resulting radiation effects. The complete diffraction-radiation problem is under development, see Finne \& Grue (1995). Relevant to most practical examples where wave drift damping is of importance, we have considered the water depth to be infinite. The formulation of the set of boundary value problems and the integral equations for the various potentials may, however, easily be extended to account for a finite water depth. The far-field forms of the potentials are somewhat modified, on the otherhand. This will also be true for final formulae for the wave drift damping coefficients.

\section{Acknowledgement}

We greatfully acknowledge the financial support by Conoco Norway, Den Norske Veritas, Saga Petroleum, and Statoil. The wave radiation-diffraction program WAMIT was provided by Massachusetts Institute of Technology and Det Norske Veritas. 


\section{Appendix A. AN INTEGRAL}

The integral (46) is given by

$$
I=\int_{S(R)}\left(\phi^{12} \frac{\partial G^{0}}{\partial n}-G^{0} \frac{\partial \phi^{12}}{\partial n}-\phi^{0} \frac{\partial G^{1}}{\partial n}+G^{1} \frac{\partial \phi^{0}}{\partial n}\right) d S
$$

By applying Green's theorem to $G^{0}$ and $2 i K \partial^{2} \phi_{I} / \partial \beta \partial K$, and to $G^{1}$ and $\phi_{I}$, and integrating over the entire free surface and $S(R)$, we obtain

$$
\int_{S_{F}+S(R)}\left(2 i K \frac{\partial^{2} \phi_{I}}{\partial \beta \partial K} \frac{\partial G^{0}}{\partial n}-2 i K G^{0} \frac{\partial}{\partial n} \frac{\partial^{2} \phi_{I}}{\partial \beta \partial K}+\phi_{I} \frac{\partial G^{1}}{\partial n}-G^{1} \frac{\partial \phi_{I}}{\partial n}\right) d S+8 \pi i K \frac{\partial^{2} \phi_{I}}{\partial \beta \partial K}=0
$$

Applying that $\partial \phi_{I} / \partial \beta=-\partial \phi_{I} / \partial \theta$, using eq. (32) for $\phi^{12}$, and (40) for $G^{1}$, and noting that the integral over the free surface in (106) vanishes, we obtain by adding (106) to the right hand side of (105)

$$
I=\int_{S(R)}\left(2 i K \frac{\partial^{2} \phi_{7}}{\partial \theta \partial K} \frac{\partial G^{0}}{\partial n}-G^{0} 2 i K \frac{\partial}{\partial n} \frac{\partial^{2} \phi_{7}}{\partial \theta \partial K}-\phi_{7} \frac{\partial G^{1}}{\partial n}+G^{1} \frac{\partial \phi_{7}}{\partial n}\right) d S+8 \pi i K \frac{\partial^{2} \phi_{I}}{\partial \beta \partial K}
$$

By then applying partial integration with respect to the $\theta$-variable, (107) reduces to

$$
I=2 i K \frac{\partial}{\partial K} \int_{S(R)}\left(\frac{\partial \phi_{7}}{\partial \theta} \frac{\partial G^{0}}{\partial R}-G^{0} \frac{\partial^{2} \phi_{7}}{\partial \theta \partial R}\right) d S+8 \pi i K \frac{\partial^{2} \phi_{I}}{\partial \beta \partial K}
$$

It may be shown by using Green's theorem that the integral over $S(R)$ in (108) is independent of the value of $R$ ( $R>$ body radius), and equals zero. The latter fact is obtained by letting $R \rightarrow \infty$ and applying the radiation conditions for $\phi_{7}$ and $G^{0}$. Thus,

$$
I=8 \pi i K \frac{\partial^{2} \phi_{I}}{\partial \beta \partial K}
$$

\section{Appendix B. A VARIANT OF STOKES' THEOREM}

A variant of Stokes' theorem reads

$$
\int_{S}(\mathbf{n} \times \nabla) \times(f \mathbf{V}) d S=\int_{C} d \mathbf{l} \times(f \mathbf{V})
$$

where $\mathbf{V}$ denotes a differentiable vector-field, f a differentiable scalar variable, and $S$ an open surface bounded by the contour $C$. The line-integral around $C$ is oriented positive with respect to the normal vector of the surface $S$. In our applications, $C$ is a contour in the mean free surface, and $S$ is assumed to have vertical walls at the mean free surface. We note that

$$
(\mathbf{n} \times \nabla) \times(f \mathbf{V})=f \mathbf{n} \times(\nabla \times \mathbf{V})+f(\mathbf{n} \cdot \nabla) \mathbf{V}-\mathbf{n} \nabla \cdot(f \mathbf{V})+(\mathbf{n} \cdot \mathbf{V}) \nabla f
$$


Applying that $\mathbf{V}=\nabla \psi$ we have $\nabla \times \mathbf{V}=0$. Noting that $d \mathbf{l} \times(f \mathbf{V})=d l\left[f\left(\mathbf{k} \psi_{n}-\mathbf{n} \psi_{z}\right)\right]$ we then obtain

$$
-\int_{S} f(\mathbf{n} \cdot \nabla) \nabla \psi d S=-\int_{S}\left[\mathbf{n} \nabla \cdot(f \nabla \psi)-\psi_{n} \nabla f\right] d S-\int_{C}\left[f\left(\mathbf{k} \psi_{n}-\mathbf{n} \psi_{z}\right)\right] d l
$$

Let $f=\phi$, and $\psi=\phi^{*}$, where $\phi^{*}$ satisfies $\nabla^{2} \phi^{*}=0$ in the fluid domain. Furthermore, let $C=C(R)$, and $S=S(R)+S(z)$, where $S(z)$ denotes a horizontal bottom with vertical coordinate $z \rightarrow-\infty$. Assuming no fluid motion at $z=-\infty$, the contribution due to integrals over $S(z)$ vanish. Exploiting the free surface boundary condition (16) for $\phi$, for a sufficiently large $R$, such that the effect of $\chi_{6}$ is negligible, i.e.

$$
\phi_{z}=K \phi+2 i \epsilon K \frac{\partial \phi}{\partial \alpha}-2 i \epsilon K \frac{\partial \phi}{\partial \theta}
$$

we find that the horizontal components of (112) read

$$
-\int_{C} K \phi \phi^{*} n_{i} d l+\int_{S}\left[\nabla \phi \cdot \nabla \phi^{*} n_{i}-\phi_{x_{i}} \phi_{n}^{*}\right] d S=\int_{S} \phi\left(\phi_{x_{i}}^{*}\right)_{n} d S-2 i K \epsilon \int_{C} \phi\left(\phi_{\beta}^{*}+\phi_{\theta}^{*}\right) n_{i} d l
$$

\section{Appendix C. THE FORCES.}

We shall evaluate the forces $\mathbf{F}_{0}$ and $B_{16}, B_{26}$, defined in Section 7. Consider first eq. (92) for $\mathbf{F}_{0}$, which reads

$$
\frac{\mathbf{F}_{0}}{\rho g A^{2}}=\frac{1}{4 K} R e \int_{S(R)}\left[\phi^{0}\left(\nabla_{h} \phi^{0 *}\right)_{n}-\phi_{x_{i}}^{0} \phi_{n}^{0 *}\right] d S
$$

By inserting the far-field form for $\phi^{0}=\phi_{I}+\phi_{7}$ we obtain

$$
\frac{\mathbf{F}_{0}}{\rho g A^{2}}=-\frac{1}{4} R e\left[\int_{0}^{2 \pi}\left|H^{0}(\theta)\right|^{2}\left(\begin{array}{c}
\cos \theta \\
\sin \theta
\end{array}\right) d \theta+\sqrt{\frac{8 \pi}{K}} H^{0 *}(\beta) e^{i \pi / 4}\left(\begin{array}{c}
\cos \beta \\
\sin \beta
\end{array}\right)\right]
$$

where we have applied the method of stationary phase to obtain the last term. (116) is a classical result and agrees with Maruo (1960), Newman (1967), and Nossen, Grue and Palm (1991).

We then consider $B_{16}, B_{26}$ which are given by eq. (93)

$$
\begin{gathered}
\frac{B_{16} \mathbf{i}+B_{26} \mathbf{j}}{\rho g A^{2}}=\operatorname{Im} \frac{1}{2}\left(\mathbf{i}_{i} \frac{\partial}{\partial \beta}-\mathbf{k} \times \mathbf{i}_{i}\right)\left[\int_{S_{F}}\left(\chi_{i}-x_{i}\right) \phi^{0} \phi_{z z}^{0 *} d S-\int_{C(R)} x_{i} \phi^{0} \phi_{n}^{0 *} d l\right] \\
-\operatorname{Re} \frac{1}{4 K} \int_{S(R)}\left[\phi^{0}\left(\nabla_{h} \phi^{1 *}\right)_{n}+\phi^{1}\left(\nabla_{h} \phi^{0 *}\right)_{n}-\phi_{n}^{0} \nabla_{h} \phi^{1 *}-\phi_{n}^{1} \nabla_{h} \phi^{0 *}\right] d S
\end{gathered}
$$

It may be shown that $\phi_{7}$ and $\phi^{13}$ have the following asymptotic forms for large $K R$

$$
\begin{aligned}
& \phi_{7}=R^{-1 / 2} H^{0}(\theta) e^{K z-i K R}\left(1+O\left((K R)^{-1}\right)\right) \\
& \phi^{13}=R^{-1 / 2} H^{13}(\theta) e^{K z-i K R}\left(1+O\left((K R)^{-1}\right)\right)
\end{aligned}
$$


where $H^{0}$ and $H^{13}$ are given by eqs. (102) and (104), respectively. The potential $\phi^{1}$ is given by

$$
\phi^{1}=2 i K \frac{\partial}{\partial K}\left(\frac{\partial \phi_{7}}{\partial \beta}+\frac{\partial \phi_{7}}{\partial \theta}\right)+\phi^{13}
$$

since $\partial \phi_{I} / \partial \beta+\partial \phi_{I} / \partial \theta=0$. For large values of $K R$ it is convenient to decompose $\phi^{1}$ by

$$
\phi^{1}=\psi^{1}+\varphi^{1}
$$

where

$$
\psi^{1}=2 K R^{1 / 2}\left(H_{\beta}^{0}+H_{\theta}^{0}\right) e^{K z-i K R}\left(1+O\left((K R)^{-1}\right)\right)
$$

and

$$
\varphi^{1}=R^{-1 / 2}\left[H^{1}+2 i K z\left(H_{\beta}^{0}+H_{\theta}^{0}\right)\right] e^{K z-i K R}\left(1+O\left((K R)^{-1}\right)\right)
$$

Here, the amplitude $H^{1}$ is defined by

$$
H^{1}=2 i K\left(H_{\beta K}^{0}+H_{\theta K}^{0}\right)+H^{13}
$$

Introducing $\mathbf{n}(\theta)=\mathbf{i} \cos \theta+\mathbf{j} \sin \theta, \mathbf{t}(\theta)=d \mathbf{n} / d \theta$, we have the following relations for the derivatives of the potentials

$$
\begin{aligned}
& \phi_{I, n}=-i K \cos (\theta-\beta) \phi_{I} \\
& \nabla_{h} \phi_{I}=-i K \phi_{I} \mathbf{n}(\beta) \\
& \left(\nabla_{h} \phi_{I}\right)_{n}=-K^{2} \cos (\theta-\beta) \phi_{I} \mathbf{n}(\beta) \\
& \phi_{7, n}=\left(-i K-\frac{1}{2 R}\right) \phi_{7}+O\left((K R)^{-5 / 2}\right) \\
& \nabla_{h} \phi_{7}=\left[\left(-i K-\frac{1}{2 R}\right) \mathbf{n}(\theta)+\frac{1}{R} \frac{H_{\theta}^{0}}{H^{0}} \mathbf{t}(\theta)\right] \phi_{7}+O\left((K R)^{-5 / 2}\right) \\
& \left(\nabla_{h} \phi_{7}\right)_{n}=\left[\left(-K^{2}+\frac{i K}{R}\right) \mathbf{n}(\theta)-\frac{i K}{R} \frac{H_{\theta}^{0}}{H^{0}} \mathbf{t}(\theta)\right] \phi_{7}+O\left((K R)^{-5 / 2}\right) \\
& \varphi_{n}^{1}=-i K \varphi^{1}+O\left((K R)^{-3 / 2}\right) \\
& \nabla_{h} \varphi^{1}=-i K \varphi^{1} \mathbf{n}(\theta)+O\left((K R)^{-3 / 2}\right) \\
& \left(\nabla_{h} \varphi^{1}\right)_{n}=-K^{2} \varphi^{1} \mathbf{n}(\theta)+O\left((K R)^{-3 / 2}\right) \\
& \psi_{n}^{1}=\left(-i K+\frac{1}{2 R}\right) \psi^{1}+O\left((K R)^{-3 / 2}\right) \\
& \nabla_{h} \psi^{1}=\left[\left(-i K+\frac{1}{2 R}\right) \psi^{1} \mathbf{n}(\theta)+\frac{1}{R} \psi_{\theta}^{1} \mathbf{t}(\theta)\right]+O\left((K R)^{-3 / 2}\right) \\
& \left(\nabla_{h} \psi^{1}\right)_{n}=\left[\left(-K^{2}-\frac{i K}{R}\right) \psi^{1} \mathbf{n}(\theta)-\frac{i K}{R} \psi_{\theta}^{1} \mathbf{t}(\theta)\right]+O\left((K R)^{-3 / 2}\right)
\end{aligned}
$$

Consider then the integral

$$
I\left(\phi^{0}, \phi^{1}\right)=-\frac{1}{4 K} R e \int_{S(R)}\left[\phi^{0}\left(\nabla_{h} \phi^{1 *}\right)_{n}+\phi^{1}\left(\nabla_{h} \phi^{0 *}\right)_{n}-\phi_{n}^{0} \nabla_{h} \phi^{1 *}-\phi_{n}^{1} \nabla_{h} \phi^{0 *}\right] d S
$$

Now, $I\left(\phi^{0}, \phi^{1}\right)=I\left(\phi^{0}, \varphi^{1}\right)+I\left(\phi^{0}, \psi^{1}\right)$. First $I\left(\phi^{0}, \varphi^{1}\right)$ is evaluated. By introducing (125) - (133) into (137) we obtain

$$
\begin{gathered}
I\left(\phi^{0}, \varphi^{1}\right)=R e \int_{S(R)} K\left[\phi_{7} \mathbf{n}(\theta)+\frac{1}{4} \phi_{I}(1+\cos (\theta-\beta))(\mathbf{n}(\beta)+\mathbf{n}(\theta))\right] \varphi^{1 *} d S \\
+O\left((K R)^{-1}\right)
\end{gathered}
$$


By carring out the vertical integration, noting that $\int_{-\infty}^{0} e^{2 K z} d z=1 /(2 K), \int_{-\infty}^{0} z e^{2 K z} d z=$ $-1 /\left(4 K^{2}\right)$, we obtain

$$
\begin{aligned}
I\left(\phi^{0}, \varphi^{1}\right)= & \frac{1}{2} R e \int_{0}^{2 \pi} H^{0}\left[H^{1 *}+i\left(H_{\beta}^{0}+H_{\theta}^{0}\right)^{*}\right] \mathbf{n}(\theta) d \theta \\
+\frac{1}{8} & R e \int_{0}^{2 \pi} R^{1 / 2} e^{i K R(1-\cos (\theta-\beta))} \times \\
& \times(1+\cos (\theta-\beta))(\mathbf{n}(\beta)+\mathbf{n}(\theta))\left[H^{1 *}+i\left(H_{\beta}^{0}+H_{\theta}^{0}\right)^{*}\right] d \theta
\end{aligned}
$$

where we have neglected terms being $O\left((K R)^{-1}\right)$. By applying the method of stationary phase to the last integral, and letting $K R \rightarrow \infty$, we obtain

$$
I\left(\phi^{0}, \varphi^{1}\right)=\frac{1}{2} R e\left[\int_{0}^{2 \pi} H^{0} H^{1 *} \mathbf{n}(\theta) d \theta+\sqrt{\frac{2 \pi}{K}} e^{i \frac{\pi}{4}} H^{1 *} \mathbf{n}(\beta)\right]+I_{1}
$$

where $I_{1}$ is expressed in terms of $\phi_{I}, \phi_{7}$, and $\psi^{1}$, i.e.

$$
I_{1}=\frac{1}{4 K} R e \int_{0}^{2 \pi} i\left[\phi_{7} \mathbf{n}(\theta)+\frac{1}{4} \phi_{I}(1+\cos (\theta-\beta))(\mathbf{n}(\beta)+\mathbf{n}(\theta))\right] \psi^{1 *} d \theta
$$

Consider then $I\left(\phi^{0}, \psi^{1}\right)$. By introducing (125) - (130) and (134) - (136) into (137), we obtain

$$
\begin{aligned}
& I\left(\phi^{0}, \psi^{1}\right)=R e \int_{S(R)} K\left[\phi_{7} \mathbf{n}(\theta)+\frac{1}{4} \phi_{I}(1+\cos (\theta-\beta))(\mathbf{n}(\beta)+\mathbf{n}(\theta))\right] \psi^{1 *} d S \\
& -R e \int_{S(R)} R^{-1} i\left[\phi_{7} \psi^{1 *} \mathbf{n}(\theta)+\frac{1}{2}\left(\phi_{7} \psi_{\theta}^{1 *}-\phi_{7, \theta} \psi^{1 *}\right) \mathbf{t}(\theta)\right] d S \\
& -\frac{1}{8} \operatorname{Re} \int_{S(R)} R^{-1} i \phi_{I} \psi^{1 *}[\mathbf{n}(\theta)+\mathbf{n}(\beta)+(1+\cos (\theta-\beta)) \mathbf{n}(\theta)] d S \\
& -\frac{1}{4} \operatorname{Re} \int_{S(R)} R^{-1} i \phi_{I} \psi_{\theta}^{1 *}[1+\cos (\theta-\beta)] \mathbf{t}(\theta) d S
\end{aligned}
$$

By then carrying out the vertical integration we find

$$
\begin{aligned}
& I\left(\phi^{0}, \psi^{1}\right)=\frac{1}{2} \operatorname{Re} \int_{C(R)}\left[\phi_{7} \mathbf{n}(\theta)+\frac{1}{4} \phi_{I}(1+\cos (\theta-\beta))(\mathbf{n}(\beta)+\mathbf{n}(\theta))\right] \psi^{1 *} R d \theta \\
& -\frac{1}{2 K} \operatorname{Re} \int_{C(R)} i\left[\phi_{7} \psi^{1 *} \mathbf{n}(\theta)+\frac{1}{2}\left(\phi_{7} \psi_{\theta}^{1 *}-\phi_{7, \theta} \psi^{1 *}\right) \mathbf{t}(\theta)\right] d \theta \\
& -\frac{1}{16 K} \operatorname{Re} \int_{C(R)} i \phi_{I} \psi^{1 *}[\mathbf{n}(\theta)+\mathbf{n}(\beta)+(1+\cos (\theta-\beta)) \mathbf{n}(\theta)] d \theta \\
& -\frac{1}{8 K} \operatorname{Re} \int_{C(R)} i \phi_{I} \psi_{\theta}^{1 *}(1+\cos (\theta-\beta)) \mathbf{t}(\theta) d \theta
\end{aligned}
$$

Consider finally the part of (117) given by

$$
I_{3}=-\frac{1}{2}\left(\mathbf{i}_{i} \frac{\partial}{\partial \beta}-\mathbf{k} \times \mathbf{i}_{i}\right) \int_{C(R)} x_{i} \operatorname{Im}\left(\phi^{0} \phi_{n}^{0 *}\right) R d \theta
$$

By partial integration we obtain

$$
I_{3}=-\frac{1}{2} \int_{C(R)} \mathbf{n}(\theta)\left(\frac{\partial}{\partial \beta}+\frac{\partial}{\partial \theta}\right) \operatorname{Im}\left(\phi^{0} \phi_{n}^{0 *}\right) R^{2} d \theta
$$


By then carrying out the differentiation with respect to the $\beta$ and $\theta$ variables, we find

$$
\begin{aligned}
& I_{3}=-\frac{1}{2} \operatorname{Re} \int_{C(R)}\left[\phi_{7} \mathbf{n}(\theta)+\frac{1}{4} \phi_{I}(1+\cos (\theta-\beta))(\mathbf{n}(\theta)+\mathbf{n}(\beta))\right] \psi^{1 *} R d \theta \\
& -\frac{1}{8} \operatorname{Re} \int_{C(R)}(\mathbf{n}(\theta)-\mathbf{n}(\beta))(1+\cos (\theta-\beta)) \phi_{I} \psi^{1 *} R d \theta \\
& -\frac{1}{8 K} \operatorname{Re} \int_{C(R)} i \phi_{I} \psi^{1 *} \mathbf{n}(\theta) d \theta
\end{aligned}
$$

Now we have that $(\mathbf{n}(\theta)-\mathbf{n}(\beta))(1+\cos (\theta-\beta))=(\mathbf{t}(\theta)+\mathbf{t}(\beta)) \sin (\theta-\beta)$. Furthermore, $K R \sin (\theta-\beta) e^{i K R(1-\cos (\theta-\beta))}=-i(d / d \theta) e^{i K R(1-\cos (\theta-\beta))}$. This means that

$$
\begin{aligned}
& -\frac{1}{8} \operatorname{Re} \int_{C(R)}(\mathbf{n}(\theta)-\mathbf{n}(\beta))(1+\cos (\theta-\beta)) \phi_{I} \psi^{1 *} R d \theta \\
& =-\frac{1}{8} \operatorname{Re} \int_{C(R)}(\mathbf{n}(\theta)-\mathbf{n}(\beta))(1+\cos (\theta-\beta)) \times \\
& \quad \times 2 K R^{3 / 2}\left(H_{\beta}^{0 *}+H_{\theta}^{0 *}\right) e^{i K R(1-\cos (\theta-\beta))} d \theta\left(1+O\left((K R)^{-1}\right)\right) \\
& =-\frac{1}{8 K} \operatorname{Re} \int_{C(R)} i \phi_{I} \psi_{\theta}^{1 *}(\mathbf{t}(\theta)+\mathbf{t}(\beta)) d \theta \\
& \quad-\frac{1}{8 K} \operatorname{Re} \int_{C(R)} i \phi_{I} \psi^{1 *} \mathbf{n}(\theta) d \theta
\end{aligned}
$$

where partial integration is applied and we have neglected terms being $O\left((K R)^{-1}\right)$. Thus, $I_{3}$ is given by

$$
\begin{aligned}
& I_{3}=-\frac{1}{2} R e \int_{C(R)}\left[\phi_{7} \mathbf{n}(\theta)+\frac{1}{4} \phi_{I}(1+\cos (\theta-\beta))(\mathbf{n}(\theta)+\mathbf{n}(\beta))\right] \psi^{1 *} R d \theta \\
& -\frac{1}{8 K} \operatorname{Re} \int_{C(R)} i \phi_{I} \psi_{\theta}^{1 *}[\mathbf{t}(\theta)+\mathbf{t}(\beta)] d \theta
\end{aligned}
$$

By then summing $I_{1}, I\left(\phi^{0}, \psi^{1}\right)$, and $I_{3}$, we obtain

$$
\begin{aligned}
& I_{1}+I\left(\phi^{0}, \psi^{1}\right)+I_{3}= \\
& \quad-\operatorname{Re}\left[\int_{0}^{2 \pi} i H^{0}\left(H_{\beta \theta}^{0}+H_{\theta \theta}^{0}\right)^{*} \mathbf{t}(\theta) d \theta+i \sqrt{\frac{2 \pi}{K}} e^{i \frac{\pi}{4}}\left(H_{\beta \theta}^{0 *}(\beta)+H_{\theta \theta}^{0 *}(\beta)\right) \mathbf{t}(\beta)\right]
\end{aligned}
$$

where the method of stationary phase is applied. The final expression for $B_{16} \mathbf{i}+B_{26} \mathbf{j}$ then becomes

$$
\begin{aligned}
& \frac{B_{16} \mathbf{i}+B_{26} \mathbf{j}}{\rho g A^{2}}=\frac{1}{2}\left(\mathbf{i}_{i} \frac{\partial}{\partial \beta}-\mathbf{k} \times \mathbf{i}_{i}\right) \int_{S_{F}}\left(\chi_{i}-x_{i}\right) \operatorname{Im}\left(\phi^{0} \phi_{z z}^{0 *}\right) d S \\
& +\frac{1}{2} \operatorname{Re} \int_{0}^{2 \pi}\left[H^{0}(\theta) H^{1 *}(\theta)\left(\begin{array}{c}
\cos \theta \\
\sin \theta
\end{array}\right)-2 i H^{0}(\theta)\left(H_{\beta \theta}^{0 *}(\theta)+H_{\theta \theta}^{0 *}(\theta)\right)\left(\begin{array}{c}
-\sin \theta \\
\cos \theta
\end{array}\right)\right] d \theta \\
& +\frac{1}{2} \operatorname{Re}\left\{\sqrt{\frac{2 \pi}{K}} e^{i \pi / 4}\left[H^{1 *}(\beta)\left(\begin{array}{c}
\cos \beta \\
\sin \beta
\end{array}\right)-2 i\left(H_{\beta \theta}^{0 *}(\beta)+H_{\theta \theta}^{0 *}(\beta)\right)\left(\begin{array}{c}
-\sin \beta \\
\cos \beta
\end{array}\right)\right]\right\}
\end{aligned}
$$




\section{References}

[1] Emmerhoff, O. J. and Sclavounos, P. D., The slow-drift motion of arrays of vertical cylinders. J. Fluid Mech. 242, 1992, 31-50.

[2] Finne, S. And Grue, J. , Analysis of the forces and the responses of floating bodies with a slow yaw-motion. 10th Int. Workshop on Water Waves and Floating Bodies, Oxford, April, 1995.

[3] Grue, J. , Wave drift damping of marine bodies in slow rotation about the vertical axis. Symposium on Waves and Nonlinear Processes in Hydrodynamics, in honour of Professor Enok Palm, Oslo, 1994.

[4] Grue, J. AND Biberg, D., Wave forces on marine structures with small speed in water of restricted depth. Appl. Ocean Res. 15, 1993, 121-135.

[5] Grue, J. AND Palm, E., Wave radiation and wave diffraction from a submerged body in a uniform current. J. Fluid Mech. 151, 1985, 257-278.

[6] Grue, J. and Palm, E., The influence of a uniform current on slowly varying forces and displacements. Appl. Ocean Res. 8, 1986, 232-239.

[7] Grue, J. and Palm, E., The mean drift force and yaw moment on marine structures in waves and current. J. Fluid Mech. 250, 1993, 121-142.

[8] Huijsmans, R.H.M. and Hermans, A.J., A fast algorithm for computation of 3-D ship motions at moderate forward speed. 4th International Confer. on Numerical Ship Hydrodynamics, 1985.

[9] Huijsmans, R.H.M., Wave drift forces in current. 16th Symp. on Naval Hydrodyn., Berkeley. Publ. by National Academy Press, Washington D.C., 1986.

[10] Maruo, H., Wave resistance of a ship in regular head sea. Bulletin of the Faculty of Engineering, Yokohama National University 9, 1960.

[11] Newman, J.N., The drift force and moment on ships in waves. J. Ship Res. 11, 1967, 51-60.

[12] Newman, J.N., Algoritms for the free-surface Green function. J. Engineering Mathematics. 19, 1985, 57-67.

[13] Newman, J.N., Wave-drift damping of floating bodies. J. Fluid Mech. 249, 1993, 241-259.

[14] Nossen, J., Grue, J. and Palm, E., Wave forces on three-dimensional floating bodies with small forward speed. J. Fluid Mech. 227, 1991, 135-160.

[15] Sclavounos, P. D., Slow-drift oscillation of compliant floating platforms. Proc. 7th Int. Confer. Behaviour of Offshore Structures (BOSS '94), MIT, ed. C. Chryssostomidis, Vol. 2, Pergamon, 1994. 
[16] Wehausen, J. V. and Laitone, E. V., Surface waves. Handbuch der Physik IX, 1960.

[17] Wichers, J.E.W. ANd Sluijs, M.F., The influence of waves on the low frequency hydrodynamic coefficients of moored vessels. Proc. Offshore Technology Conference, Houston, OTC 3625, 1979.

[18] Wichers, J.E.W. and Huijsmans, R.H.M., On the low frequency hydrodynamic damping forces acting on offshore moored vessels. Proc. Offshore Technology Conference, Houston, OTC 4831, 1984.

[19] Zhao, R., Faltinsen, O.M., Krokstad, J.R. and Aanesland, V., Wavecurrent Interaction Effects on Large-Volume Structures. Proc. 5th Int. Confer. Behaviour of Offshore Structures (BOSS '88), Trondheim, ed. T. Moan, N. Janby \& O. M. Faltinsen, Vol. 2, Tapir Publishers, 1988.

[20] ZhaO, R. AND Faltinsen, O.M., Interaction between current, waves and marine structures. 5th Int. Conf. on Num. Ship Hydrodynamics, Hiroshima, National Academy Press, Washington D. C., 1989. 